\title{
RADIAL AVERAGING OPERATOR ACTING ON BERGMAN AND LEBESGUE SPACES
}

\author{
TANELI KORHONEN, JOSÉ ÁNGEL PELÁEZ, AND JOUNI RÄTTYÄ
}

ABSTRACT. It is shown that the radial averaging operator

$$
T_{\omega}(f)(z)=\frac{\int_{|z|}^{1} f\left(s \frac{z}{|z|}\right) \omega(s) d s}{\widehat{\omega}(z)}, \quad \widehat{\omega}(z)=\int_{|z|}^{1} \omega(s) d s,
$$

induced by a radial weight $\omega$ on the unit disc $\mathbb{D}$, is bounded from the weighted Bergman space $A_{\nu}^{p}$, where $0<p<\infty$ and the radial weight $\nu$ satisfies $\widehat{\nu}(r) \leqslant C \widehat{\nu}\left(\frac{1+r}{2}\right)$ for all $0 \leqslant r<1$, to $L_{\nu}^{p}$ if and only if the self-improving condition $\sup _{0 \leqslant r<1} \frac{\hat{\omega}(r)^{p}}{\int_{r}^{1} s \nu(s) d s} \int_{0}^{r} \frac{t \nu(t)}{\hat{\omega}(t)^{p}} d t<\infty$ is satisfied. Further, two characterizations of the weak type inequality

$$
\eta\left(\left\{z \in \mathbb{D}:\left|T_{\omega}(f)(z)\right| \geqslant \lambda\right\}\right) \lesssim \lambda^{-p}\|f\|_{L_{\nu}^{p}}^{p}, \quad \lambda>0,
$$

are established for arbitrary radial weights $\omega, \nu$ and $\eta$. Moreover, differences and interrelationships between the cases $A_{\nu}^{p} \rightarrow L_{\nu}^{p}, L_{\nu}^{p} \rightarrow L_{\nu}^{p}$ and $L_{\nu}^{p} \rightarrow L_{\nu}^{p, \infty}$ are analyzed.

\section{INTRODUCTION AND MAIN RESULTS}

A function $\omega: \mathbb{D} \rightarrow[0, \infty)$, integrable over the unit disc $\mathbb{D}$, is called a weight. It is radial if $\omega(z)=\omega(|z|)$ for all $z \in \mathbb{D}$. For $0<p<\infty$ and a weight $\omega$, the Lebesgue space $L_{\omega}^{p}$ consists of complex-valued measurable functions $f$ in $\mathbb{D}$ such that

$$
\|f\|_{L_{\omega}^{p}}=\left(\int_{\mathbb{D}}|f(z)|^{p} \omega(z) d A(z)\right)^{\frac{1}{p}}<\infty
$$

where $d A(z)=\frac{d x d y}{\pi}$ denotes the element of the normalized Lebesgue area measure on $\mathbb{D}$. The weighted Bergman space $A_{\omega}^{p}$ is the space of analytic functions in $L_{\omega}^{p}$.

For a radial weight $\omega$, we assume throughout the paper that $\widehat{\omega}(z)=\int_{|z|}^{1} \omega(s) d s>0$ for all $z \in \mathbb{D}$, otherwise the Bergman space $A_{\omega}^{p}$ would contain all analytic functions in $\mathbb{D}$. For a radial weight, the norm convergence in the Hilbert space $A_{\omega}^{2}$ implies the uniform convergence on compact subsets of $\mathbb{D}$ and so the point evaluations are bounded linear functionals on $A_{\omega}^{2}$. Therefore there exist reproducing Bergman kernels $B_{z}^{\omega} \in A_{\omega}^{2}$ such that

$$
f(z)=\left\langle f, B_{z}^{\omega}\right\rangle_{A_{\omega}^{2}}=\int_{\mathbb{D}} f(\zeta) \overline{B_{z}^{\omega}(\zeta)} \omega(\zeta) d A(\zeta), \quad z \in \mathbb{D}, \quad f \in A_{\omega}^{2} .
$$

The Hilbert space $A_{\omega}^{2}$ is a closed subspace of $L_{\omega}^{2}$, and hence the orthogonal Bergman projection from $L_{\omega}^{2}$ to $A_{\omega}^{2}$ is given by

$$
P_{\omega}(f)(z)=\int_{\mathbb{D}} f(\zeta) \overline{B_{z}^{\omega}(\zeta)} \omega(\zeta) d A(\zeta), \quad z \in \mathbb{D} .
$$

A radial weight $\omega$ belongs to the class $\widehat{\mathcal{D}}$ if there exists a constant $C=C(\omega)>1$ such that the doubling condition $\widehat{\omega}(r) \leqslant C \widehat{\omega}\left(\frac{1+r}{2}\right)$ is satisfied for all $0 \leqslant r<1$. Moreover, if there exist

Date: December 3, 2018.

Key words and phrases. Inner function, Bergman space, Hardy space, Radial average operator, Schwarz-Pick lemma, doubling weight, Muckenhoupt weight, maximal function, Carleson measure.

This research was supported in part by Ministerio de Economía y Competitivivad, Spain, projects MTM201452865-P and MTM2017-90584-REDT; La Junta de Andalucía, project FQM210; Academy of Finland project no. 268009, and by Faculty of Science and Forestry of University of Eastern Finland. 
$K=K(\omega)>1$ and $C=C(\omega)>1$ such that

$$
\widehat{\omega}(r) \geqslant C \widehat{\omega}\left(1-\frac{1-r}{K}\right), \quad 0 \leqslant r<1,
$$

then we write $\omega \in \check{\mathcal{D}}$. The intersection $\widehat{\mathcal{D}} \cap \breve{\mathcal{D}}$ is denoted by $\mathcal{D}$. The definitions of these classes of weights are of geometric nature, and the classes themselves arise naturally in the study of classical operators. For example, it is known that the Bergman projection $P_{\omega}$ induced by a radial weight $\omega$ is bounded from $L^{\infty}$ of $\mathbb{D}$ to the Bloch space $\mathcal{B}$ if and only if $\omega \in \widehat{\mathcal{D}}$, and further, $P_{\omega}: L^{\infty} \rightarrow \mathcal{B}$ is bounded and onto if and only if $\omega \in \mathcal{D}$ [15. A radial weight $\omega$ is regular if $\widehat{\omega}(r)=\omega(r)(1-r)$ for all $0 \leqslant r<1$. The class of regular weights is denoted by $\mathcal{R}$, and $\mathcal{R} \subsetneq \mathcal{D}$. For basic properties of these classes of weights and more, see [9, 10, 15] and the references therein.

This paper concerns the radial (Hardy) averaging operator $T_{\omega}$, induced by a radial weight $\omega$, and defined by

$$
T_{\omega}(f)(z)=\frac{\int_{|z|}^{1} f\left(s \frac{z}{|z|}\right) \omega(s) d s}{\widehat{\omega}(z)}, \quad z \in \mathbb{D} \backslash\{0\},
$$

and its maximal version

$$
T_{\omega}^{N}(f)(z)=\frac{\int_{|z|}^{1} N(f)\left(s \frac{z}{|z|}\right) \omega(s) d s}{\widehat{\omega}(z)}, \quad z \in \mathbb{D} \backslash\{0\},
$$

where $N(f)(z)=\sup _{\zeta \in \Gamma(z)}|f(\zeta)|$ is the maximal function on the non-tangential approach region

$$
\Gamma(z)=\left\{\xi \in \mathbb{D}:|\theta-\arg (\xi)|<\frac{1}{2}\left(1-\frac{|\xi|}{r}\right)\right\}, \quad z=r e^{i \theta} \in \overline{\mathbb{D}} \backslash\{0\},
$$

with vertex in the punctured closed unit disc.

Hardy averaging operators have been extensively studied since decades ago in the context of Lebesgue spaces, see [1, 2, 3, 6, 8, 17] and the references therein. The question of when $T_{\omega}: L_{\nu}^{p} \rightarrow L_{\eta}^{p}$ is bounded can be answered by a classical result due to Muckenhoupt [8]. Namely, the condition

$$
M_{p}=M_{p}(\omega, \nu, \eta)=\sup _{0<r<1}\left(\int_{r}^{1}\left(\frac{\omega(s)}{s \nu(s)}\right)^{p^{\prime}} s \nu(s) d s\right)^{\frac{1}{p^{\prime}}}\left(\int_{0}^{r} \frac{\eta(s) s}{\widehat{\omega}(s)^{p}} d s\right)^{\frac{1}{p}}<\infty
$$

being a sufficient condition for $T_{\omega}: L_{\nu}^{p} \rightarrow L_{\eta}^{p}$ to be bounded and an estimate from above for the operator norm follow by applying [8, Theorem 2], and a testing similar to that in the proof of the said theorem gives the necessity and a lower bound for the operator norm. We state this result for further reference as follows.

Theorem A. Let $1<p<\infty$ and $\omega, \nu$ and $\eta$ radial weights. Then $T_{\omega}: L_{\nu}^{p} \rightarrow L_{\eta}^{p}$ is bounded if and only if $M_{p}(\omega, \nu, \eta)<\infty$. Moreover, $M_{p} \leqslant\left\|T_{\omega}\right\|_{L_{\nu}^{p} \rightarrow L_{\eta}^{p}} \leqslant p(p-1)^{\frac{1-p}{p}} M_{p}$.

It is worth noticing that under the hypotheses $\omega, \nu \in \mathcal{R}$ and $\eta \in \breve{\mathcal{D}}$ the averaging operator $T_{\omega}$ and the maximal Bergman projection

$$
P_{\omega}^{+}(f)(z)=\int_{\mathbb{D}} f(\zeta)\left|B_{z}^{\omega}(\zeta)\right| \omega(\zeta) d A(\zeta), \quad z \in \mathbb{D},
$$

are simultaneously bounded from $L_{\nu}^{p}$ to $L_{\eta}^{p}$ by [7. Moreover, in the particular case $\omega \equiv 1$ with $\nu \in \mathcal{R}, T_{\omega}: A_{\nu}^{p} \rightarrow L_{\nu}^{p}$ is bounded if and only if the Bergman projection $P_{\omega}$ is bounded on $L_{\nu}^{p}$ [16]. Therefore the averaging operator $T_{\omega}$, which is interesting in its own right, is closely related to the weighted Bergman projection $P_{\omega}$ and it might be further used as a model for its study. With this aim and that of studying the difference respect to their action on Lebesgue spaces, we consider the averaging operator $T_{\omega}$ acting from $A_{\nu}^{p}$ to $L_{\nu}^{p}$ when $\nu \in \widehat{\mathcal{D}}$. 
We recall that a positive Borel measure $\mu$ on $\mathbb{D}$ is a $q$-Carleson measure for $A_{\nu}^{p}$ if the identity operator is bounded from $A_{\nu}^{p}$ to $L_{\mu}^{q}$. The $q$-Carleson measures for $A_{\nu}^{p}$ induced by $\nu \in \widehat{\mathcal{D}}$ are described in [12]. By denoting

$$
d \mu_{p, \omega, \nu}(z)=\widehat{\omega}(z)^{p-1} \omega(z)\left(\int_{0}^{|z|} \frac{\nu(s)}{\widehat{\omega}(s)^{p}} s d s\right) d A(z), \quad z \in \mathbb{D},
$$

we state the first main result of the paper as follows.

Theorem 1. Let $0<p<\infty, \nu \in \widehat{\mathcal{D}}$ and $\omega$ a radial weight. Then the following statements are equivalent:

(i) $T_{\omega}^{N}: A_{\nu}^{p} \rightarrow L_{\nu}^{p}$ is bounded;

(ii) $T_{\omega}: A_{\nu}^{p} \rightarrow L_{\nu}^{p}$ is bounded;

(iii) $\widehat{\mathcal{D}}_{p}(\omega, \nu)=\sup _{0 \leqslant r<1} \frac{\hat{\omega}(r)^{p}}{\int_{r}^{1} s \nu(s) d s} \int_{0}^{r} \frac{t \nu(t)}{\hat{\omega}(t)^{p}} d t<\infty$;

(iv) $\mu_{p, \omega, \nu}$ is a q-Carleson measure for $A_{\nu}^{q}$ for some (equivalently for each) $0<q<\infty$. Moreover,

$$
\left\|T_{\omega}^{N}\right\|_{A_{\nu}^{p} \rightarrow L_{\nu}^{p}}^{p}=\left\|T_{\omega}\right\|_{A_{\nu}^{p} \rightarrow L_{\nu}^{p}}^{p}=\|I d\|_{A_{\nu}^{p} \rightarrow L_{\mu_{p, \omega}, \nu}^{p}}^{p}=\widehat{\mathcal{D}}_{p}(\omega, \nu) .
$$

To obtain the necessary condition $\widehat{\mathcal{D}}_{p}(\omega, \nu)<\infty$, we observe first that indicators cannot be used to build test functions, as it happens in Theorem $\mathrm{A}$. This is a significant difference between the analytic and the classical case. We overcome this obstacle by using derivatives of Bergman reproducing kernels $B_{a}^{\nu}$ as test functions. The asymptotic behavior of $\left\|\left(B_{a}^{\nu}\right)^{(N)}\right\|_{A_{\nu}^{p}}$ is well understood by the recent study [13] and these estimates are strongly in use in our reasoning. In addition, we need lower estimates for the $L_{\nu}^{p}$-norm of $T_{\omega}\left(\left(B_{a}^{\nu}\right)^{(N)}\right)$. We prove these estimates by using Hardy-Littlewood inequalities and smooth polynomials related to Hadamard products. This approach actually enables us to obtain a sharp necessary condition for the boundedness in a much more general situation, see Proposition 6 below. As an immediate consequence of these deductions we observe that $T_{\omega}: L_{\nu}^{p} \rightarrow L_{\nu}^{q}$ fails to be bounded if $q>p$ and $\nu \in \widehat{\mathcal{D}}$. Another pivotal dissimilarity between the analytic and the classical case consists of finding an appropriate way to obtain a sufficient condition in the former one. It turns out that Carleson measures is an adequate one as is already seen in the statement. We will also show that the condition $\widehat{\mathcal{D}}_{p}(\omega, \nu)<\infty$ is self-improving in the sense that if $\widehat{\mathcal{D}}_{p}(\omega, \nu)<\infty$, then there exists $\varepsilon=\varepsilon(p, \omega, \nu)>0$ such that $\widehat{\mathcal{D}}_{p-\varepsilon}(\omega, \nu)<\infty$, and this observation will play an important role in the proof.

By using Theorems 1 and $\mathrm{A}$ along with [13, Theorem 11], we establish the following result.

Corollary 2. Let $1<p<\infty$ and $\omega, \nu \in \mathcal{R}$. Then the following statements are equivalent:

(i) $T_{\omega}: A_{\nu}^{p} \rightarrow L_{\nu}^{p}$ is bounded;

(ii) $T_{\omega}: L_{\nu}^{p} \rightarrow L_{\nu}^{p}$ is bounded;

(iii) $P_{\omega}^{+}: L_{\nu}^{p} \rightarrow L_{\nu}^{p}$ is bounded;

(iv) $P_{\omega}: L_{\nu}^{p} \rightarrow L_{\nu}^{p}$ is bounded.

It is not a surprise that the statement in Corollary 2 fails without any local regularity hypotheses on the weights because $\omega$ being absolutely continuous with respect to $\nu$ is a necessary condition for $T_{\omega}$ to be bounded on $L_{\nu}^{p}$ by Theorem $\mathrm{A}$, see Corollary 10 below.

We also consider the $(p, p)$-weak type inequality for the radial averaging operator. Our main result in this direction is the following.

Theorem 3. Let $1<p<\infty$ and $\omega, \nu, \eta$ be radial weights. Then the following statements are equivalent:

(i) $T_{\omega}: L_{\nu}^{p} \rightarrow L_{\eta}^{p, \infty}$ is bounded;

(ii) $N_{p}(\omega, \nu, \eta)=\sup _{0 \leqslant t<r<1}\left(\frac{\int_{t}^{r} \eta(s) s d s}{\widehat{\omega}(t)^{p}}\right)^{\frac{1}{p}}\left(\int_{r}^{1}\left(\frac{\omega(s)}{s \nu(s)}\right)^{p^{\prime}} s \nu(s) d s\right)^{\frac{1}{p^{\prime}}}<\infty$; 
(iii) $M_{p, \varepsilon}=M_{p, \varepsilon}(\omega, \nu, \eta)=\sup _{0 \leqslant r<1}\left(\widehat{\omega}(r)^{\varepsilon} \int_{0}^{r} \frac{s \eta(s)}{\widehat{\omega}(s)^{p+\varepsilon}} d s\right)^{\frac{1}{p}}\left(\int_{r}^{1}\left(\frac{\omega(s)}{s \nu(s)}\right)^{p^{\prime}} s \nu(s) d s\right)^{\frac{1}{p^{\prime}}}<$ $\infty$ for some (equivalently for each) $\varepsilon>0$.

Moreover, for each fixed $\varepsilon>0$,

$$
\left\|T_{\omega}\right\|_{L_{\nu}^{p} \rightarrow L_{\eta}^{p, \infty}}=N_{p}(\omega, \nu, \eta) \asymp M_{p, \varepsilon}(\omega, \nu, \eta) .
$$

A calculation shows that Theorem A can be deduced from the corresponding strong inequality for the simple case $\omega \equiv 1$ by modifying the weights involved. However, this is no longer true for weak-type inequalities due to the nature of the level sets for $\left|T_{\omega}\right|$. On the other hand, although conditions similar to Theorem 3(ii) appear frequently in the literature [2, 6, conditions analogous to Theorem 3(iii), which is a kind of generalization of the classical result of Andersen and Muckenhoupt [1, Theorem 2], seem to have been less explored. Therefore, we believe that Part (iii) in Theorem 3 adds theoretical and practical value to the result. As for the proof of Theorem [3, it follows the leading idea of that of [1, Theorem 2] but a good number of non-trivial modifications are needed.

It is also worth noticing that for each $1<p<\infty$ there are weights $(\omega, \nu, \eta)$, such that $T_{\omega}: L_{\nu}^{p} \rightarrow L_{\eta}^{p, \infty}$ is bounded but $T_{\omega}: L_{\nu}^{p} \rightarrow L_{\eta}^{p}$ is not. Nonetheless, as a byproduct of our results we deduce the following.

Corollary 4. Let $1<p<\infty, \omega \in \widehat{\mathcal{D}}$ and $\nu \in \mathcal{D}$. Then $T_{\omega}: L_{\nu}^{p} \rightarrow L_{\nu}^{p}$ bounded if and only if $T_{\omega}: A_{\nu}^{p} \rightarrow L_{\nu}^{p}$ bounded and $T_{\omega}: L_{\nu}^{p} \rightarrow L_{\nu}^{p, \infty}$ bounded.

In Section 2 we prove Theorem 1 and Corollary 2, Theorem 3 is proved in Section 3 , where we also provide the details of the proof of Corollary 4 and offer concrete examples related to the results obtained in this study.

Throughout the paper $\frac{1}{p}+\frac{1}{p^{\prime}}=1$ for $1<p<\infty$. Further, the letter $C=C(\cdot)$ will denote an absolute constant whose value depends on the parameters indicated in the parenthesis, and may change from one occurrence to another. We will use the notation $a \lesssim b$ if there exists a constant $C=C(\cdot)>0$ such that $a \leqslant C b$, and $a \gtrsim b$ is understood in an analogous manner. In particular, if $a \lesssim b$ and $a \gtrsim b$, then we will write $a=b$. An observant reader notices that this last notation is already in use in (1.2) and (1.3).

\section{Analytic Case}

2.1. Test functions. We first establish necessary conditions for $T_{\omega}: A_{\nu}^{p} \rightarrow L_{\eta}^{q}$ to be bounded when $0<p \leqslant q<\infty, \nu \in \widehat{\mathcal{D}}$ and $\omega, \eta$ are radial weights. To do this, some background material is needed.

For a radial weight $\omega$, the normalized monomials $\frac{z^{n}}{\sqrt{2 \int_{0}^{1} r^{2 n+1} \omega(r) d r}}, n \in \mathbb{N} \cup\{0\}$, form the standard orthonormal basis of $A_{\omega}^{2}$, and hence

$$
B_{z}^{\omega}(\zeta)=\sum_{n=0}^{\infty} \frac{(\zeta \bar{z})^{n}}{2 \int_{0}^{1} r^{2 n+1} \omega(r) d r}, \quad z, \zeta \in \mathbb{D} .
$$

Write

$$
\omega_{t, x}=\int_{t}^{1} s^{x} \omega(s) d s, \quad 0 \leqslant x<\infty, \quad 0 \leqslant t<1,
$$

and $\omega_{x}=\omega_{0, x}$ for short. For $f \in \mathcal{H}(\mathbb{D})$ with Maclaurin series $f(z)=\sum_{n=0}^{\infty} \hat{f}(n) z^{n}$ and $k \in \mathbb{N} \cup\{0\}$, denote $\Delta_{k} f(z)=\sum_{n=2^{k}}^{2^{k+1}-1} \hat{f}(n) z^{n}$. With these preparations we can state and prove the following mean estimate for the image of $\left(B_{a}^{\nu}\right)^{(N)}$ under $T_{\omega}$.

Lemma 5. Let $0<q<\infty, \nu \in \hat{\mathcal{D}}, \omega$ a radial weight, $N \in \mathbb{N}$ and $a \in \mathbb{D}$ with $|a| \geqslant 1-\frac{1}{2 N}$. Then

$$
\widehat{\omega}(t)^{q} M_{q}^{q}\left(t, T_{\omega}\left(\left(B_{a}^{\nu}\right)^{(N)}\right)\right) \gtrsim \begin{cases}\frac{\widehat{\omega}(a)^{q}}{\widehat{\nu}(a)^{q}(1-|a|)^{q(N+1)-1}}, & 0 \leqslant t \leqslant|a|, \\ \frac{\hat{\omega}(t)^{q}}{\widehat{\nu}(a)^{q}(1-|a|)^{q(N+1)-1}}, & |a| \leqslant t<1 .\end{cases}
$$


Proof. First observe that

$$
\begin{aligned}
\widehat{\omega}(t) T_{\omega}\left(\left(B_{a}^{\nu}\right)^{(N)}\right)\left(t e^{i \theta}\right) & =\int_{t}^{1}\left(B_{a}^{\nu}\right)^{(N)}\left(s e^{i \theta}\right) \omega(s) d s \\
& =\int_{t}^{1}\left(\sum_{j=N}^{\infty} \frac{j(j-1) \cdots(j-N+1)\left(s e^{i \theta}\right)^{j-N} \bar{a}^{j}}{2 \nu_{2 j+1}}\right) \omega(s) d s \\
& =\sum_{j=N}^{\infty} \frac{j(j-1) \cdots(j-N+1) e^{i \theta(j-N)} \bar{a}^{j}}{2 \nu_{2 j+1}} \omega_{t, j-N} .
\end{aligned}
$$

From now on we split the proof into four cases. Let first $0 \leqslant t \leqslant|a|$ and $0<q \leqslant 2$. Then the classical Hardy-Littlewood inequality [4, Theorem 6.2] gives

$$
\widehat{\omega}(t)^{q} M_{q}^{q}\left(t, T_{\omega}\left(\left(B_{a}^{\nu}\right)^{(N)}\right)\right) \gtrsim \sum_{j=N}^{\infty} \frac{j^{N q+q-2} \omega_{t, j-N}^{q}|a|^{j q}}{\nu_{2 j+1}^{q}} .
$$

Choose $N^{\star} \in \mathbb{N}$ such that $1-\frac{1}{N^{\star}} \leqslant|a|<1-\frac{1}{N^{\star}+1}$. Then the inequality $0 \leqslant t \leqslant|a|$ and [9, Lemma 2.1(iv)] yield

$$
\begin{aligned}
\sum_{j=N}^{N^{\star}} \frac{j^{N q+q-2} \omega_{t, j-N}^{q}|a|^{j q}}{\nu_{2 j+1}^{q}} & \gtrsim \sum_{j=N}^{N^{\star}} \frac{j^{N q+q-2} \omega_{|a|, j-N}^{q}}{\nu_{2 j+1}^{q}} \gtrsim \widehat{\omega}(a)^{q} \sum_{j=N}^{N^{\star}} \frac{j^{N q+q-2}}{\widehat{\nu}\left(1-\frac{1}{2 j+1}\right)^{q}} \\
& \gtrsim \widehat{\omega}(a)^{q} \int_{N}^{N^{\star}+1} \frac{s^{q(N+1)-2}}{\widehat{\hat{\nu}\left(1-\frac{1}{2 s+1}\right)^{q}}} d s=\widehat{\omega}(a)^{q} \int_{N}^{N^{\star}+1} \frac{s^{q(N+1)-2}}{\widehat{\nu}\left(1-\frac{1}{s}\right)^{q}} d s \\
& \geqslant \widehat{\omega}(a)^{q} \int_{1-\frac{1}{N}}^{1-\frac{1}{N^{\star}+1}} \frac{d t}{\widehat{\nu}(t)^{q}(1-t)^{q(N+1)}} \\
& \geqslant \widehat{\omega}(a)^{q} \int_{1-\frac{1}{N}}^{|a|} \frac{d t}{\widehat{\nu}(t)^{q}(1-t)^{q(N+1)}} \\
& \geqslant \widehat{\omega}(a)^{q} \int_{2|a|-1}^{|a|} \frac{d t}{\widehat{\nu}(t)^{q}(1-t)^{q(N+1)}} \\
& \gtrsim \frac{\widehat{\omega}(a)^{q}}{\widehat{\nu}(2|a|-1)^{q}(1-|a|)^{q(N+1)-1}} \\
& \gtrsim \frac{\widehat{\omega}(a)^{q}}{\widehat{\nu}(a)^{q}(1-|a|)^{q(N+1)-1}},
\end{aligned}
$$

which combined with (2.4) gives the assertion in the case $0 \leqslant t \leqslant|a|$ and $0<q \leqslant 2$. If $|a| \leqslant t<1$, then the assertion readily follows by applying the estimate $\omega_{t, j-N} \gtrsim \widehat{\omega}(t)$ in the reasoning (2.5).

Let now $q>2$ (this approach actually works for any $q>1$ ). We begin by showing that

$$
\left\|\Delta_{k} F_{N+1}\right\|_{H^{q}}^{q} \gtrsim 2^{k[q(N+1)-1]}, \quad k \geqslant \log _{2} N,
$$

where $F_{N+1}$ is the function, analytic in the unit disc, defined by the Maclaurin series

$$
F_{N+1}(z)=\sum_{j=N}^{\infty} j(j-1) \cdots(j-N+1) z^{j}, \quad z \in \mathbb{D},
$$

and thus

$$
\Delta_{k} F_{N+1}(z)=\sum_{j=2^{k}}^{2^{k+1}-1} j(j-1) \cdots(j-N+1) z^{j}, \quad z \in \mathbb{D} .
$$


By using the well known estimate $M_{\infty}(r, f) \lesssim(\rho-r)^{-\frac{1}{q}} M_{q}(\rho, f)$, valid for all $0<r<\rho<1$, $0<q<\infty$ and $f \in \mathcal{H}(\mathbb{D})$, and [11, Lemma 10], we deduce

$$
\begin{aligned}
M_{\infty}\left(1-\frac{1}{2^{k}}, \Delta_{k} F_{N+1}\right) & \lesssim\left(\frac{1}{2^{k}}-\frac{1}{2^{k+1}-1}\right)^{-\frac{1}{q}} M_{q}\left(1-\frac{1}{2^{k+1}-1}, \Delta_{k} F_{N+1}\right) \\
& \approx 2^{\frac{k}{q}}\left\|\Delta_{k} F_{N+1}\right\|_{H^{q}},
\end{aligned}
$$

which together with (2.7) gives

$$
\begin{aligned}
&\left\|\Delta_{k} F_{N+1}\right\|_{H^{q}}^{q} \gtrsim 2^{-k} M_{\infty}^{q}\left(1-\frac{1}{2^{k}}, \Delta_{k} F_{N+1}\right)=2^{-k}\left(\sum_{j=2^{k}}^{2^{k+1}-1} j^{N}\left(1-\frac{1}{2^{k}}\right)^{j}\right)^{q} \\
& \geqslant 2^{N k q-k}\left(2^{k}\left(1-2^{-k}\right)^{2^{k+1}}\right)^{q}=2^{k[q(N+1)-1]} .
\end{aligned}
$$

Therefore (2.6) is now proved.

Let $0 \leqslant t \leqslant|a|$. For $|a| \geqslant 1 / 2$, choose $k \in \mathbb{N}$ such that $1-2^{-k} \leqslant|a|<1-2^{-k-1}$. Then (2.3), [13, Lemma D] and [9, Lemma 2.1] yield

$$
\begin{aligned}
\widehat{\omega}(t)^{q} M_{q}^{q}\left(t, T_{\omega}\left(\left(B_{a}^{\nu}\right)^{N}\right)\right. & \left.\left.\gtrsim \int_{0}^{2 \pi}\right|_{j=2^{k}} ^{2^{k+1}-1} \frac{j(j-1) \cdots(j-N+1) e^{i \theta(j-N)} \bar{a}^{j}}{2 \nu_{2 j+1}} \omega_{t, j-N}\right|^{q} d \theta \\
& \left.\left.\gtrsim \frac{|a|^{q 2^{k+1}} \omega_{t, 2^{k+1}-N}^{q}}{\nu_{2^{k+1}}^{q}} \int_{0}^{2 \pi}\right|_{j=2^{k}} ^{\mid 2^{k+1}-1} j(j-1) \cdots(j-N+1) e^{i \theta(j-N)}\right|^{q} d \theta \\
& \gtrsim \frac{\omega_{|a| 2^{k+1}-N}^{q}}{\widehat{\nu}\left(1-\frac{1}{2^{k+1}}\right)^{q}}\left\|\Delta_{k} F_{n+1}\right\|_{H^{q}}^{q} \gtrsim \frac{\widehat{\omega}(a)^{q}}{\widehat{\nu}(a)^{q}} 2^{k[(N+1) q-1]} \\
& =\frac{\widehat{\omega}(a)^{q}}{\widehat{\nu}(a)^{q}(1-|a|)^{q(N+1)-1}},
\end{aligned}
$$

and thus the assertion for $0 \leqslant t \leqslant|a|$ and $q>2$ is valid. The assertion in the case $t \geqslant|a|$ and $q>2$ readily follows by applying the estimate $\omega_{t, 2^{k+1}-N} \gtrsim \widehat{\omega}(t)$ in the reasoning above.

Proposition 6. Let $0<p \leqslant q<\infty, \nu \in \widehat{\mathcal{D}}$ and $\omega, \eta$ radial weights. If $T_{\omega}: A_{\nu}^{p} \rightarrow L_{\eta}^{q}$ is bounded, then

$$
\sup _{0 \leqslant r<1} \frac{\widehat{\omega}(r)^{q}}{(1-r)^{\frac{q}{p}-1}\left(\int_{r}^{1} s \nu(s) d s\right)^{\frac{q}{p}}} \int_{0}^{r} \frac{t \eta(t)}{\widehat{\omega}(t)^{q}} d t \lesssim\left\|T_{\omega}\right\|_{A_{\nu}^{p} \rightarrow L_{\eta}^{q}}^{q}<\infty
$$

and

$$
\sup _{0 \leqslant r<1} \frac{\left.\int_{r}^{1} s \eta(s) d s\right)}{(1-r)^{\frac{q}{p}-1}\left(\int_{r}^{1} s \nu(s) d s\right)^{\frac{q}{p}}} \lesssim\left\|T_{\omega}\right\|_{A_{\nu}^{p} \rightarrow L_{\eta}^{q}}^{q}<\infty .
$$

Proof. Assume that $T_{\omega}: A_{\nu}^{p} \rightarrow L_{\eta}^{q}$ is bounded. Then, for each $a \in \mathbb{D}$ and $N \in \mathbb{N}$,

$$
\begin{aligned}
\left\|T_{\omega}\left(\left(B_{a}^{\nu}\right)^{(N)}\right)\right\|_{L_{\eta}^{q}}^{q} & \leqslant\left\|T_{\omega}\right\|_{A_{\nu}^{p} \rightarrow L_{\eta}^{q}}^{q}\left\|\left(B_{a}^{\nu}\right)^{(N)}\right\|_{A_{\nu}^{p}}^{q} \\
& =\left\|T_{\omega}\right\|_{A_{\nu}^{p} \rightarrow L_{\eta}^{q}}^{q}\left(\int_{0}^{|a|} \frac{d t}{\widehat{\nu}(t)^{p-1}(1-t)^{p(N+1)}}\right)^{\frac{q}{p}}, \quad|a| \rightarrow 1^{-},
\end{aligned}
$$

by [13, Theorem 1 ]. If $p \geqslant 1$, then

$$
\left\|\left(B_{a}^{\nu}\right)^{(N)}\right\|_{A_{\nu}^{p}}^{p} \lesssim \frac{1}{\widehat{\nu}(a)^{p-1}} \int_{0}^{|a|} \frac{d t}{(1-t)^{p(N+1)}}=\frac{1}{\widehat{\nu}(a)^{p-1}(1-|a|)^{p(N+1)-1}}, \quad|a| \rightarrow 1^{-},
$$


for each $N \in \mathbb{N}$. If $0<p<1$, choose $N=N(p, \nu) \in \mathbb{N}$ such that $N>\frac{1+(1-p) \beta}{p}-1$, where $\beta=\beta(\nu)>0$ is that of [9, Lemma 2.1(ii)]. Then

$$
\begin{aligned}
\left\|\left(B_{a}^{\nu}\right)^{(N)}\right\|_{A_{\nu}^{p}}^{p} & \lesssim \frac{1}{\widehat{\nu}(a)^{p-1}(1-|a|)^{(1-p) \beta}} \int_{0}^{|a|} \frac{d t}{(1-t)^{p(N+1)-(1-p) \beta}} \\
& =\frac{1}{\widehat{\nu}(a)^{p-1}(1-|a|)^{p(N+1)-1}}, \quad|a| \rightarrow 1^{-} .
\end{aligned}
$$

That is, for each $0<p<\infty$, there exists $N=N(p, \nu) \in \mathbb{N}$ such that

$$
\left\|\left(B_{a}^{\nu}\right)^{(N)}\right\|_{A_{\nu}^{p}}^{p} \lesssim \frac{1}{\widehat{\nu}(a)^{p-1}(1-|a|)^{p(N+1)-1}}, \quad|a| \rightarrow 1^{-} .
$$

On the other hand, Lemma 5 yields

$$
\begin{aligned}
\left\|T_{\omega}\left(\left(B_{a}^{\nu}\right)^{(N)}\right)\right\|_{L_{\eta}^{q}}^{q} & =\int_{0}^{|a|} M_{q}^{q}\left(t, T_{\omega}\left(B_{a}^{\nu}\right)^{(N)}\right) t \eta(t) d t+\int_{|a|}^{1} M_{q}^{q}\left(t, T_{\omega}\left(B_{a}^{\nu}\right)^{(N)}\right) t \eta(t) d t \\
\gtrsim & \left(\int_{0}^{|a|} \frac{t \eta(t)}{\widehat{\omega}(t)^{q}} d t\right) \frac{\widehat{\omega}(a)^{q}}{\widehat{\nu}(a)^{q}(1-|a|)^{q(N+1)-1}} \\
& +\frac{\widehat{\eta}(a)}{\widehat{\nu}(a)^{q}(1-|a|)^{q(N+1)-1}}, \quad|a| \geqslant 1-\frac{1}{2 N} .
\end{aligned}
$$

The assertions follow by combining (2.10), (2.11) and (2.12).

It is worth noticing that replacing the derivatives of the Bergman reproducing kernels by the monomials in the proof of Proposition 6 yields analogous conditions to (2.8) and (2.9) but without the factor $(1-r)^{\frac{q}{p}-1}$ in the denominator on the left hand side. So, one gets the same condition for $q=p$ but a weaker one for $q>p$.

We make two observations on Proposition [6. First, (2.8) implies (2.9) if $\omega \in \widehat{\mathcal{D}}$ and $\eta \in \breve{\mathcal{D}}$. Namely, by [9, Lemma 2.1] there exists $\alpha=\alpha(\omega, q)>0$ such that $(1-t)^{\alpha} \widehat{\omega}(t)^{-q}$ is essentially decreasing, and hence

$$
\int_{0}^{r} \frac{t \eta(t)}{\widehat{\omega}(t)^{q}} d t \gtrsim \frac{1}{\widehat{\omega}(r)^{q}} \int_{0}^{r}\left(\frac{1-r}{1-t}\right)^{\alpha} t \eta(t) d t
$$

Let $K=K(\eta)>1$ be that of the definition of $\check{\mathcal{D}}$, and define $r_{n}=1-K^{-n}$ for all $n \in \mathbb{N} \cup\{0\}$. If $r_{1} \leqslant r<1$, then there exists $m \in \mathbb{N}$ such that $r_{m} \leqslant r<r_{m+1}$. Therefore

$$
\begin{aligned}
\int_{0}^{r}\left(\frac{1-r}{1-t}\right)^{\alpha} t \eta(t) d t & \geqslant \int_{r_{m-1}}^{r_{m}}\left(\frac{1-r}{1-t}\right)^{\alpha} t \eta(t) d t \geqslant r_{1}\left(\frac{1-r_{m+1}}{1-r_{m-1}}\right)^{\alpha}\left(\widehat{\eta}\left(r_{m-1}\right)-\widehat{\eta}\left(r_{m}\right)\right) \\
& \geqslant r_{1} \frac{(C-1)}{K^{2 \alpha}} \widehat{\eta}\left(r_{m}\right) \geqslant r_{1} \frac{(C-1)}{K^{2 \alpha}} \widehat{\eta}(r), \quad r_{1} \leqslant r<1,
\end{aligned}
$$

for all $\alpha>0$. So, if $\omega \in \widehat{\mathcal{D}}$ and $\eta \in \check{\mathcal{D}}$, then

$$
\int_{0}^{r} \frac{t \eta(t)}{\widehat{\omega}(t)^{q}} d t \gtrsim \frac{\widehat{\eta}(r)}{\widehat{\omega}(r)^{q}}, \quad r_{1}(\eta)=r_{1} \leqslant r<1,
$$

and thus (2.9) follows from (2.8) in this case.

Second, if $\nu \in \hat{\mathcal{D}}$, the supremum in (2.9) is finite if and only if $A_{\nu}^{p}$ is continuously embedded into $L_{\eta}^{q}$ by [12, Theorem 1], and thus $\left\|I_{d}\right\|_{A_{\nu}^{p} \rightarrow L_{\eta}^{q}} \lesssim\left\|T_{\omega}\right\|_{A_{\nu}^{p} \rightarrow L_{\eta}^{q}}$ for each radial weight $\omega$.

Bearing in mind the special case $\eta=\nu$ of $(2.9)$, we get the following immediate consequence.

Corollary 7. Let $0<p<q<\infty$ and $\nu \in \hat{\mathcal{D}}$. Then $T_{\omega}: A_{\nu}^{p} \rightarrow L_{\nu}^{q}$ is unbounded for each radial weight $\omega$. 


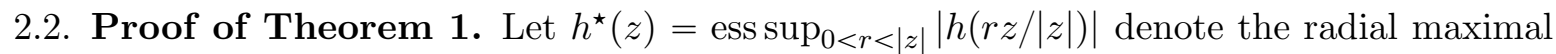
function of $h$ at $z \in \mathbb{D} \backslash\{0\}$.

Lemma 8. Let $0<p \leqslant 1 \leqslant q<\infty$ and $\omega$ a radial weight. Then there exist constants $C_{1}=C_{1}(p, \omega)>0$ and $C_{2}=C_{2}(q, \omega)>0$ such that

$$
\left(\int_{r}^{1} h\left(t e^{i \theta}\right) \omega(t) d t\right)^{p} \leqslant C_{1} \int_{r}^{1} h^{\star}\left(t e^{i \theta}\right)^{p} \widehat{\omega}(t)^{p-1} \omega(t) t d t
$$

and

$$
\int_{r}^{1} h\left(t e^{i \theta}\right)^{q} \widehat{\omega}(t)^{q-1} \omega(t) d t \leqslant C_{2}\left(\int_{r}^{1} h^{\star}\left(t e^{i \theta}\right) \omega(t) t d t\right)^{q}
$$

for all non-negative measurable functions $h$ on $\mathbb{D}$ and $\theta \in \mathbb{R}$.

Proof. For $1<K<\infty$ and $0 \leqslant r<1$, define $\rho_{n}=\rho_{n}(\omega, K, r)=\min \{0 \leqslant t<1: \widehat{\omega}(t)=$ $\left.\widehat{\omega}(r) K^{-n}\right\}$ for all $n \in \mathbb{N}$, and set $\rho_{0}=r$. Then $\left\{\rho_{n}\right\}$ is increasing such that $\rho_{n} \rightarrow 1^{-}$, as $n \rightarrow \infty$, and

$$
\int_{\rho_{n}}^{\rho_{n+1}} \omega(t) d t=\widehat{\omega}\left(\rho_{n}\right)-\widehat{\omega}\left(\rho_{n+1}\right)=\widehat{\omega}\left(\rho_{j}\right) K^{j-n}\left(\frac{K-1}{K}\right), \quad n, j \in \mathbb{N} \cup\{0\} .
$$

Hence

$$
\begin{aligned}
\left(\int_{r}^{1} h\left(t e^{i \theta}\right) \omega(t) d t\right)^{p} & =\left(\sum_{n=0}^{\infty} \int_{\rho_{n}}^{\rho_{n+1}} h\left(t e^{i \theta}\right) \omega(t) d t\right)^{p} \\
& \leqslant\left(\sum_{n=0}^{\infty} h^{\star}\left(\rho_{n+1} e^{i \theta}\right)\left(\widehat{\omega}\left(\rho_{n}\right)-\widehat{\omega}\left(\rho_{n+1}\right)\right)\right)^{p} \\
& =(K-1)^{p}\left(\sum_{n=0}^{\infty} h^{\star}\left(\rho_{n+1} e^{i \theta}\right) \widehat{\omega}\left(\rho_{n+1}\right)\right)^{p} \\
& \leqslant(K-1)^{p} \sum_{n=0}^{\infty} h^{\star}\left(\rho_{n+1} e^{i \theta}\right)^{p} \widehat{\omega}\left(\rho_{n+1}\right)^{p} \frac{\widehat{\omega}\left(\rho_{n+1}\right)-\widehat{\omega}\left(\rho_{n+2}\right)}{\widehat{\omega}\left(\rho_{n+1}\right) \frac{K-1}{K}} \\
& =K(K-1)^{p-1} \sum_{n=0}^{\infty} h^{\star}\left(\rho_{n+1} e^{i \theta}\right)^{p} \widehat{\omega}\left(\rho_{n+1}\right)^{p-1}\left(\widehat{\omega}\left(\rho_{n+1}\right)-\widehat{\omega}\left(\rho_{n+2}\right)\right) \\
& \leqslant K(K-1)^{p-1} \sum_{n=0}^{\infty} \int_{\rho_{n+1}}^{\rho_{n+2}} h^{\star}\left(t e^{i \theta}\right)^{p} \widehat{\omega}(t)^{p-1} \omega(t) d t \\
& \leqslant \frac{K(K-1)^{p-1}}{\rho_{1}} \int_{r}^{1} h^{\star}\left(t e^{i \theta}\right)^{p} \widehat{\omega}(t)^{p-1} \omega(t) t d t .
\end{aligned}
$$


In a similar manner one deduces

$$
\begin{aligned}
\int_{r}^{1} h\left(t e^{i \theta}\right)^{q} \widehat{\omega}(t)^{q-1} \omega(t) d t & =\sum_{n=0}^{\infty} \int_{\rho_{n}}^{\rho_{n+1}} h\left(t e^{i \theta}\right)^{q} \widehat{\omega}(t)^{q-1} \omega(t) d t \\
& \leqslant \sum_{n=0}^{\infty} h^{\star}\left(\rho_{n+1} e^{i \theta}\right)^{q} \widehat{\omega}\left(\rho_{n}\right)^{q-1} \int_{\rho_{n}}^{\rho_{n+1}} \omega(t) d t \\
& =\frac{K-1}{K} \sum_{n=0}^{\infty} h^{\star}\left(\rho_{n+1} e^{i \theta}\right)^{q} \widehat{\omega}\left(\rho_{n}\right)^{q} \\
& \leqslant \frac{K-1}{K}\left(\sum_{n=0}^{\infty} h^{\star}\left(\rho_{n+1} e^{i \theta}\right) \widehat{\omega}\left(\rho_{n}\right)\right)^{q} \\
& =\frac{K-1}{K}\left(\sum_{n=0}^{\infty} h^{\star}\left(\rho_{n+1} e^{i \theta}\right) \int_{\rho_{n+1}}^{\rho_{n+2}} \omega(t) d t \frac{\widehat{\omega}\left(\rho_{n}\right)}{\widehat{\omega}\left(\rho_{n}\right) \frac{K-1}{K^{2}}}\right)^{q} \\
& \leqslant \frac{(K-1)^{1-q}\left(\sum_{n=0}^{\infty} \int_{\rho_{n+1}}^{\rho_{n+2}} h^{\star}\left(t e^{i \theta}\right) \omega(t) d t\right)^{q}}{} \\
& \leqslant K^{2 q-1}(K-1)^{1-q}\left(\frac{1}{\rho_{1}} \int_{\rho_{1}}^{1} h^{\star}\left(t e^{i \theta}\right) \omega(t) t d t\right)^{q} \\
& \leqslant \frac{K^{2 q-1}(K-1)^{1-q}}{\rho_{1}^{q}}\left(\int_{r}^{1} h^{\star}\left(t e^{i \theta}\right) \omega(t) t d t\right)^{q}
\end{aligned}
$$

and thus the lemma is proved.

The next lemma shows that the condition $\widehat{\mathcal{D}}_{p}(\omega, \nu)<\infty$ is self-improving in the sense that if it is satisfied for some $p>0$, then it is also satisfied when $p$ is replaced by a slightly smaller number.

Lemma 9. Let $0<p<\infty$ and $\nu, \omega$ radial weights on $\mathbb{D}$. Then

$$
\widehat{\mathcal{D}}_{p}(\omega, \nu) \leqslant \widehat{\mathcal{D}}_{p-\varepsilon}(\omega, \nu) \leqslant \frac{p}{p-\varepsilon\left(1+\widehat{\mathcal{D}}_{p}(\omega, \nu)\right)} \widehat{\mathcal{D}}_{p}(\omega, \nu)
$$

for all $\varepsilon \in\left(0, \frac{p}{\hat{\mathcal{D}}_{p}(\omega, \nu)+1}\right)$.

Proof. The first inequality is obvious. Let us denote $\nu_{1}(t)=t \nu(t)$. An integration by parts gives

$$
\begin{aligned}
\int_{0}^{r} \frac{\nu_{1}(t)}{\widehat{\omega}(t)^{p-\varepsilon}} d t & =\widehat{\omega}(r)^{\varepsilon} \int_{0}^{r} \frac{\nu_{1}(t)}{\widehat{\omega}(t)^{p}} d t+\varepsilon \int_{0}^{r}\left(\int_{0}^{s} \frac{\nu_{1}(t)}{\widehat{\omega}(t)^{p}} d t\right) \widehat{\omega}(s)^{\varepsilon-1} \omega(s) d s \\
& \leqslant \widehat{\mathcal{D}}_{p}(\omega, \nu) \frac{\widehat{\nu_{1}}(r)}{\widehat{\omega}(r)^{p-\varepsilon}}+\varepsilon \widehat{\mathcal{D}}_{p}(\omega, \nu) \int_{0}^{r} \frac{\widehat{\nu_{1}}(s)}{\widehat{\omega}(s)^{p+1-\varepsilon}} \omega(s) d s
\end{aligned}
$$


and since Fubini's theorem yields

$$
\begin{aligned}
\int_{0}^{r} \frac{\widehat{\nu_{1}}(s)}{\widehat{\omega}(s)^{p+1-\varepsilon}} \omega(s) d s & =\int_{0}^{r} \frac{\int_{s}^{1} \nu_{1}(t) d t}{\widehat{\omega}(s)^{p+1-\varepsilon}} \omega(s) d s \\
& =\int_{0}^{r} \frac{\int_{s}^{r} \nu_{1}(t) d t}{\widehat{\omega}(s)^{p+1-\varepsilon}} \omega(s) d s+\widehat{\nu_{1}}(r) \int_{0}^{r} \frac{\omega(s)}{\widehat{\hat{\omega}(s)^{p+1-\varepsilon}}} d s \\
& =\int_{0}^{r} \nu_{1}(t)\left(\int_{0}^{t} \frac{\omega(s)}{\widehat{\omega}(s)^{p+1-\varepsilon}} d s\right) d t+\widehat{\nu_{1}}(r) \int_{0}^{r} \frac{\omega(s)}{\widehat{\hat{\omega}(s)^{p+1-\varepsilon}}} d s \\
& =\frac{1}{p-\varepsilon}\left(\int_{0}^{r} \frac{\nu_{1}(t)}{\widehat{\omega}(t)^{p-\varepsilon}} d t-\frac{1}{\widehat{\omega}(0)^{p-\varepsilon}} \int_{0}^{r} \nu_{1}(t) d t+\frac{\widehat{\nu_{1}}(r)}{\widehat{\hat{\omega}(r)^{p-\varepsilon}}}-\frac{\widehat{\nu_{1}}(r)}{\widehat{\hat{\omega}(0)^{p-\varepsilon}}}\right) \\
& \leqslant \frac{1}{p-\varepsilon}\left(\int_{0}^{r} \frac{\nu_{1}(t)}{\widehat{\omega}(t)^{p-\varepsilon}} d t+\frac{\widehat{\nu_{1}}(r)}{\widehat{\omega}(r)^{p-\varepsilon}}\right),
\end{aligned}
$$

we deduce

$$
\int_{0}^{r} \frac{\nu_{1}(t)}{\widehat{\omega}(t)^{p-\varepsilon}} d t \leqslant \frac{\widehat{\mathcal{D}}_{p}(\omega, \nu)\left(1+\frac{\varepsilon}{p-\varepsilon}\right)}{1-\frac{\hat{\mathcal{D}}_{p}(\omega, \nu) \varepsilon}{p-\varepsilon}} \frac{\widehat{\nu_{1}}(r)}{\widehat{\omega}(r)^{p-\varepsilon}}=\frac{p \widehat{\mathcal{D}}_{p}(\omega, \nu)}{p-\varepsilon\left(1+\widehat{\mathcal{D}}_{p}(\omega, \nu)\right)} \frac{\widehat{\nu_{1}}(r)}{\widehat{\omega}(r)^{p-\varepsilon}}
$$

for $\varepsilon \in\left(0, \frac{p}{\widehat{\mathcal{D}}_{p}(\omega, \nu)+1}\right)$. The assertion follows.

With these preparations we are ready for the proof.

Proof of Theorem 1. Obviously, (i) implies (ii), and (ii) implies (iii) together with the inequality $\widehat{\mathcal{D}}_{p}(\omega, \nu) \lesssim\left\|T_{\omega}\right\|_{A_{\nu}^{p} \rightarrow L_{\nu}^{p}}^{p}$ follows from the case $q=p$ of Proposition 6 .

By [12, Theorem 1], $\mu_{p, \omega, \nu}$ is a $q$-Carleson measure for $A_{\nu}^{q}$ if and only if $\mu_{p, \omega, \nu}(S(a)) \lesssim$ $\nu(S(a))$ for all Carleson squares $S(a)=\left\{z \in \mathbb{D}:|\arg z-\arg a|<\frac{1-|a|}{2},|z| \geqslant 1-|a|\right\}$ with $a \in \mathbb{D} \backslash\{0\}$. But Fubini's theorem yields

$$
\begin{aligned}
\int_{|a|}^{1} \widehat{\omega}(t)^{p-1} \omega(t) \int_{0}^{t} \frac{\nu(s) s d s}{\widehat{\omega}(s)^{p}} d t= & \int_{|a|}^{1} \widehat{\omega}(t)^{p-1} \omega(t)\left(\int_{0}^{|a|}+\int_{|a|}^{t}\right) \frac{\nu(s) s d s}{\widehat{\omega}(s)^{p}} d t \\
= & \left(\int_{|a|}^{1} \widehat{\omega}(t)^{p-1} \omega(t) d t\right) \int_{0}^{|a|} \frac{\nu(s) s d s}{\widehat{\omega}(s)^{p}} \\
& +\int_{|a|}^{1} \frac{\nu(s) s}{\widehat{\omega}(s)^{p}} \int_{s}^{1} \widehat{\omega}(t)^{p-1} \omega(t) d t d s \\
= & \frac{\widehat{\omega}(a)^{p}}{p} \int_{0}^{|a|} \frac{\nu(s) s d s}{\widehat{\omega}(s)^{p}}+\frac{1}{p} \int_{|a|}^{1} \nu(s) s d s,
\end{aligned}
$$

and it follows that (iii) and (iv) are equivalent.

Assume (iii), and let first $0<p \leqslant 1$. By Lemma 8 and Fubini's theorem,

$$
\begin{aligned}
\int_{0}^{1}\left(T_{\omega}^{N}(f)\left(r e^{i \theta}\right)\right)^{p} \nu(r) r d r & =\int_{0}^{1}\left(\int_{r}^{1} N(f)\left(t e^{i \theta}\right) \omega(t) d t\right)^{p} \frac{\nu(r) r}{\widehat{\omega}(r)^{p}} d r \\
& \lesssim \int_{0}^{1}\left(\int_{r}^{1}(N(f))^{\star}\left(t e^{i \theta}\right)^{p} \widehat{\omega}(t)^{p-1} \omega(t) t d t\right) \frac{\nu(r) r}{\widehat{\omega}(r)^{p}} d r \\
& =\int_{0}^{1} N(f)^{p}\left(t e^{i \theta}\right) \widehat{\omega}(t)^{p-1} \omega(t)\left(\int_{0}^{t} \frac{\nu(r) r}{\widehat{\omega}(r)^{p}} d r\right) t d t
\end{aligned}
$$


An integration with respect to $\theta$ and the Hardy-Littlewood maximal theorem [5. Theorem 3.1, p. 57] (or [10, Lemma 4.4]) together with (iii) now yield

$$
\begin{aligned}
\left\|T_{\omega}^{N}(f)\right\|_{L_{\nu}^{p}}^{p} & \lesssim \int_{\mathbb{D}}|f(z)|^{p} \widehat{\omega}(z)^{p-1} \omega(z)\left(\int_{0}^{|z|} \frac{\nu(r) r}{\widehat{\omega}(r)^{p}} d r\right) d A(z) \\
& =\int_{\mathbb{D}}|f(z)|^{p} d \mu_{p, \omega, \nu}(z) \leqslant\|I d\|_{A_{\nu}^{p} \rightarrow L_{\mu_{p, \omega}, \nu}^{p}}^{p}\|f\|_{A_{\nu}^{p}}^{p}
\end{aligned}
$$

Thus $T_{\omega}^{N}: A_{\omega}^{p} \rightarrow L_{\omega}^{p}$ is bounded. Moreover, the estimates above together with [14, Theorem 3] and (2.14) give

$$
\|T\|_{A_{\nu}^{p} \rightarrow L_{\nu}^{p}}^{p} \leqslant\left\|T^{N}\right\|_{A_{\nu}^{p} \rightarrow L_{\nu}^{p}}^{p} \lesssim\|I d\|_{A_{\nu}^{p} \rightarrow L_{\mu p, \omega, \nu}^{p}}^{p}=\widehat{\mathcal{D}}_{p}(\omega, \nu)
$$

provided $0<p \leqslant 1$.

Let now $1<p<\infty$, and fix $\varepsilon=\varepsilon(p, \omega, \nu) \in\left(0, \min \left\{p-1, \frac{p}{\widehat{\mathcal{D}}_{p}(\omega, \nu)}\right\}\right)$. Define $h(z)=$ $\omega(z)^{\frac{1}{p^{\prime}}} \widehat{\omega}(z)^{\frac{-p+1+\varepsilon}{p}}$ for all $z \in \mathbb{D}$, and set $\Omega=\{z \in \mathbb{D}: \omega(z)>0\}$. Then Hölder's inequality, Fubini's theorem and the Hardy-Littlewood maximal theorem yield

$$
\begin{aligned}
\left\|T_{\omega}^{N}(f)\right\|_{L_{\nu}^{p}}^{p} & =\int_{\mathbb{D}}\left(\int_{|z|}^{1} N(f)\left(t \frac{z}{|z|}\right) \omega(t) \chi_{\Omega}(t) d t\right)^{p} \frac{\nu(z)}{\widehat{\omega}(z)^{p}} d A(z) \\
& =\int_{\mathbb{D}}\left(\int_{|z|}^{1} N(f)\left(t \frac{z}{|z|}\right) \frac{\omega(t)}{h(t)} h(t) \chi_{\Omega}(t) d t\right)^{p} \frac{\nu(z)}{\widehat{\omega}(z)^{p}} d A(z) \\
& \leqslant \int_{\mathbb{D}}\left(\int_{|z|}^{1} N^{p}(f)\left(t \frac{z}{|z|}\right)\left(\frac{\omega(t)}{h(t)}\right)^{p} \chi_{\Omega}(t) d t\right)\left(\int_{|z|}^{1} h(s)^{p^{\prime}} d s\right)^{p-1} \frac{\nu(z)}{\widehat{\omega}(z)^{p}} d A(z) \\
& =\int_{0}^{1} \int_{0}^{2 \pi}\left(\int_{r}^{1} N^{p}(f)\left(t e^{i \theta}\right)\left(\frac{\omega(t)}{h(t)}\right)^{p} \chi_{\Omega}(t) d t\right)\left(\int_{r}^{1} h(s)^{p^{\prime}} d s\right)^{p-1} \frac{\nu(r)}{\widehat{\omega}(r)^{p}} r d \theta d r \\
& =\int_{0}^{1} \int_{0}^{2 \pi} N^{p}(f)\left(t e^{i \theta}\right) d \theta\left(\frac{\omega(t)}{h(t)}\right)^{p} \chi_{\Omega}(t) \int_{0}^{t}\left(\int_{r}^{1} h(s)^{p^{\prime}} d s\right)^{p-1} \frac{\nu(r)}{\widehat{\omega}(r)^{p}} r d r d t \\
& \lesssim \int_{0}^{1} \int_{0}^{2 \pi}\left|f\left(t e^{i \theta}\right)\right|^{p} d \theta\left(\frac{\omega(t)}{h(t)}\right)^{p} \chi_{\Omega}(t) \int_{0}^{t}\left(\int_{r}^{1} h(s)^{p^{\prime}} d s\right)^{p-1} \frac{\nu(r)}{\widehat{\omega}(r)^{p}} r d r d t \\
& =\int_{\mathbb{D}}|f(z)|^{p}\left(\frac{\omega(z)}{h(z)}\right)^{p} \chi_{\Omega}(z) \int_{0}^{|z|}\left(\int_{r}^{1} h(s)^{p^{\prime}} d s\right)^{p-1} \frac{\nu(r)}{\widehat{\omega}(r)^{p}} r d r d A(z) \\
& =\left(\frac{p-1}{\varepsilon}\right)^{p-1} \int_{\mathbb{D}}|f(z)|^{p} \omega(z) \widehat{\omega}(z)^{p-1-\varepsilon} \int_{0}^{|z|} \frac{\nu(r)}{\widehat{\omega}(r)^{p-\varepsilon}} r d r d A(z) \\
& \lesssim \int_{\mathbb{D}}|f(z)|^{p} d \mu_{p-\varepsilon, \omega, \nu}(z) .
\end{aligned}
$$

Now that $\mu_{p-\varepsilon, \omega, \nu}$ is a $p$-Carleson measure for $A_{\omega}^{p}$ by (2.14) and Lemma 9, it follows that $T_{\omega}^{N}: A_{\nu}^{p} \rightarrow L_{\nu}^{p}$ is bounded. Moreover, a reasoning similar to that in the case $0<p \leqslant 1$ together with Lemma 9 gives

$$
\left\|T_{\omega}\right\|_{A_{\nu}^{p} \rightarrow L_{\nu}^{p}}^{p} \leqslant\left\|T_{\omega}^{N}\right\|_{A_{\nu}^{p} \rightarrow L_{\nu}^{p}}^{p} \lesssim\|I d\|_{A_{\nu}^{p} \rightarrow L_{\mu_{p-\varepsilon, \omega, \nu}}^{p}}^{p}=\widehat{\mathcal{D}}_{p-\varepsilon}(\omega, \nu) \asymp \widehat{\mathcal{D}}_{p}(\omega, \nu) .
$$

The proof is complete.

Proof of Corollary Q2. Since $\omega, \nu \in \mathcal{R}$, the result follows joining [13, Theorem 11], Theorem $\mathrm{A}$ and Theorem 1 . 


\section{WEAK TYPE INEQUALITIES}

We use the conventions $0 \cdot \infty=0$ and $1 / 0=\infty$. The next proof follows ideas from [1]. Proof of Theorem 3. Assume first (i), that is,

$$
\lambda^{p} \eta\left(\left\{z \in \mathbb{D}:\left|T_{\omega}(f)(z)\right|>\lambda\right\}\right) \lesssim\|f\|_{L_{\nu}^{p}}^{p}, \quad \lambda>0, \quad f \in L_{\nu}^{p} .
$$

Let $h(r)=\left(\int_{r}^{1}\left(\frac{\omega(s)}{s \nu(s)}\right)^{p^{\prime}} s \nu(s) d s\right)^{\frac{1}{p^{\prime}}}$ and denote

$$
N(t, r)=\left(\frac{\int_{t}^{r} \eta(s) s d s}{\widehat{\omega}(t)^{p}}\right)^{\frac{1}{p}}\left(\int_{r}^{1}\left(\frac{\omega(s)}{s \nu(s)}\right)^{p^{\prime}} s \nu(s) d s\right)^{\frac{1}{p^{\prime}}}, \quad 0 \leqslant t<r<1,
$$

for short. Let us rule out the trivial cases $h(r)=0$ or $h(r)=\infty$ first. If $h(r)=0$, then $N(t, r)=0$ for all $0 \leqslant t<r$ by the convention. If $h(r)=\infty$, then $\left(\omega(s \nu)^{-1}\right)^{1 / p} \notin L_{\omega}^{p^{\prime}}(r, 1)$, and hence there exists $g \in L_{\omega}^{p}(r, 1)$ such that $g\left(\omega(s \nu)^{-1}\right)^{1 / p} \notin L_{\omega}^{1}(r, 1)$. Define $f(z)=$ $g(|z|)\left(\omega(z)(|z| \nu(z))^{-1}\right)^{\frac{1}{p}}$ for $r \leqslant|z|<1$, and let $f \equiv 0$ elsewhere on $\mathbb{D}$. Then $T_{\omega}(f)(z)=\infty$ for $|z| \leqslant r$, and therefore the weak inequality (3.1) yields

$$
\begin{aligned}
\int_{0}^{r} s \eta(s) d s & \leqslant \int_{\left\{z \in \mathbb{D}:\left|T_{\omega}(f)(z)\right|>\lambda\right\}} \eta(z) d A(z) \\
& \lesssim \frac{1}{\lambda^{p}} \int_{\{z \in \mathbb{D}: r \leqslant|z|<1\}}|f(z)|^{p} \nu(z) d A(z)=\frac{2}{\lambda^{p}} \int_{r}^{1}|g(s)|^{p} \omega(s) d s, \quad \lambda>0 .
\end{aligned}
$$

By letting $\lambda \rightarrow \infty$, we deduce $\int_{0}^{r} \eta(s) s d s=0$, and thus $N(t, r)=0$ for all $0 \leqslant t<r$ in this case also.

Assume that $0<h(r)<\infty$, and let $f_{r}(z)=\left(\frac{\omega(z)}{|z| \nu(z)}\right)^{p^{\prime} / p} \chi_{\mathbb{D} \backslash D(0, r)}(z), z \in \mathbb{D}$, and

$$
\lambda_{r, t}=\frac{\int_{r}^{1}\left(\frac{\omega(s)}{s \nu(s)}\right)^{p^{\prime}} s \nu(s) d s}{\widehat{\omega}(t)}, \quad 0 \leqslant t \leqslant r<1 .
$$

Then

$$
\left\|f_{r}\right\|_{L_{\nu}^{p}}^{p}=2 \int_{r}^{1}\left(\frac{\omega(s)}{s \nu(s)}\right)^{p^{\prime}} \nu(s) s d s
$$

and

$$
\begin{aligned}
T_{\omega}\left(f_{r}\right)(z) & =\widehat{\omega}(z)^{-1} \int_{|z|}^{1} \chi_{[r, 1)}(s)\left(\frac{\omega(s)}{s \nu(s)}\right)^{p^{\prime} / p} \omega(s) d s \\
& =\widehat{\omega}(z)^{-1} \int_{r}^{1}\left(\frac{\omega(s)}{s \nu(s)}\right)^{p^{\prime}} s \nu(s) d s \geqslant \lambda_{r, t}, \quad 0 \leqslant t<|z| \leqslant r<1 .
\end{aligned}
$$

Therefore

$$
\begin{aligned}
\int_{t}^{r} \eta(s) s d s & \leqslant 2 \eta\left(\left\{z \in \mathbb{D}:\left|T_{\omega}\left(f_{r}\right)(z)\right| \geqslant \lambda_{r, t}\right\}\right) \leqslant 2\left\|T_{\omega}\right\|_{L_{\nu}^{p} \rightarrow L_{\eta}^{p, \infty}}^{p} \frac{\left\|f_{r}\right\|_{L_{\nu}^{p}}^{p}}{\lambda_{r, t}^{p}} \\
& =\left\|T_{\omega}\right\|_{L_{\nu}^{p} \rightarrow L_{\eta}^{p, \infty}}^{p} \frac{\widehat{\omega}(t)^{p} \int_{r}^{1}\left(\frac{\omega(s)}{s \nu(s)}\right)^{p^{\prime}} s \nu(s) d s}{\left(\int_{r}^{1}\left(\frac{\omega(s)}{s \nu(s)}\right)^{p^{\prime}} s \nu(s) d s\right)^{p}} \\
& =\left\|T_{\omega}\right\|_{L_{\nu}^{p} \rightarrow L_{\eta}^{p, \infty}}^{p} \frac{\widehat{\omega}(t)^{p}}{\left(\int_{r}^{1}\left(\frac{\omega(s)}{s \nu(s)}\right)^{p^{\prime}} s \nu(s) d s\right)^{\frac{p}{p^{\prime}}}}
\end{aligned}
$$


and thus

$$
\frac{\int_{t}^{r} \eta(s) s d s}{\widehat{\omega}(t)^{p}}\left(\int_{r}^{1}\left(\frac{\omega(s)}{s \nu(s)}\right)^{p^{\prime}} s \nu(s) d s\right)^{p / p^{\prime}} \lesssim\left\|T_{\omega}\right\|_{L_{\nu}^{p} \rightarrow L_{\eta}^{p, \infty}}^{p}, \quad 0 \leqslant t<r<1 .
$$

This implies (ii) and $N_{p}(\omega, \nu, \eta) \lesssim\left\|T_{\omega}\right\|_{L_{\nu}^{p} \rightarrow L_{\eta}^{p, \infty}}$.

Assume next (ii). Then, for each $\varepsilon>0$, we have

$$
\begin{aligned}
N_{p}^{p}(\omega, \nu, \eta) \frac{1}{\varepsilon}\left(\frac{1}{\widehat{\omega}(r)^{\varepsilon}}-\frac{1}{\widehat{\omega}(0)^{\varepsilon}}\right) & =N_{p}^{p}(\omega, \nu, \eta) \int_{0}^{r} \frac{\omega(t)}{\widehat{\omega}(t)^{1+\varepsilon}} d t \\
& \geqslant h^{p}(r) \int_{0}^{r}\left(\int_{t}^{r} \eta(s) s d s\right) \frac{\omega(t)}{\widehat{\omega}(t)^{1+\varepsilon+p}} d t \\
& =h^{p}(r) \int_{0}^{r}\left(\int_{0}^{s} \frac{\omega(t)}{\widehat{\omega}(t)^{1+\varepsilon+p}} d t\right) \eta(s) s d s \\
& =\frac{h^{p}(r)}{p+\varepsilon} \int_{0}^{r}\left(\frac{1}{\widehat{\omega}(s)^{\varepsilon+p}}-\frac{1}{\widehat{\omega}(0)^{\varepsilon+p}}\right) \eta(s) s d s
\end{aligned}
$$

from which (iii) and the inequality $N_{p}(\omega, \nu, \eta) \gtrsim M_{p, \varepsilon}(\omega, \nu, \eta)$ for each fixed $\varepsilon>0$ follow.

Assume now (iii), and let $\varepsilon>0$. Let $f$ be a compactly supported non-negative step function on $\mathbb{D}$, that is, $f=\sum_{j=1}^{l} P_{j} \chi_{R_{j}}$, where $P_{j} \geqslant 0$ and $R_{j}=\left\{r e^{i \theta}: 0 \leqslant A_{j} \leqslant r \leqslant B_{j}<1, c_{j} \leqslant \theta \leqslant\right.$ $\left.d_{j}\right\}$ with $d_{j}-c_{j} \leqslant 2 \pi$. Define $E_{\theta}(\lambda)=\left\{r \in(0,1): T_{\omega}(f)\left(r e^{i \theta}\right)>\lambda\right\}$ for any $\theta \in[0,2 \pi)$ and $\lambda>0$, and $H(r)=\int_{0}^{r} \frac{s \eta(s)}{\hat{\omega}(s)^{p+\varepsilon}} d s$ for all $0 \leqslant r<1$. If $r_{0}=\inf \{r \in(0,1): h(r)<\infty\}>0$, then $\eta(r)=0$ almost everywhere on $\left[0, r_{0}\right]$. Therefore $\int_{E_{\theta}(\lambda)} \eta(s) d s=\int_{E_{\theta}(\lambda) \cap\left(r_{0}, 1\right)} \eta(s) d s$, where

$$
E_{\theta}(\lambda) \cap\left(r_{0}, 1\right)=\bigcup_{k=1}^{n}\left(a_{k}, b_{k}\right), \quad r_{0} \leqslant a_{1}<\cdots<b_{k} \leqslant a_{k+1}<\cdots<b_{n}<1 .
$$

Let $r \in\left[a_{k}, b_{k}\right]$ for some $k=1, \ldots, n$. Then $\widehat{\omega}(r) \leqslant \lambda^{-1} \int_{r}^{1} f\left(s e^{i \theta}\right) \omega(s) d s$, and Hölder's inequality yields

$$
\begin{aligned}
\widehat{\omega}(r)^{p} & \leqslant \lambda^{-p}\left(\int_{r}^{1} f\left(s e^{i \theta}\right) h(s)^{1 / p}(s \nu(s))^{1 / p} h(s)^{-1 / p} \frac{\omega(s)}{(s \nu(s))^{1 / p}} d s\right)^{p} \\
& \leqslant \lambda^{-p} \int_{r}^{1} f\left(s e^{i \theta}\right)^{p} h(s) s \nu(s) d s\left(\int_{r}^{1} h(s)^{-\frac{1}{p-1}}\left(\frac{\omega(s)}{s \nu(s)}\right)^{p^{\prime}} s \nu(s) d s\right)^{p-1} \\
& =\lambda^{-p} \int_{r}^{1} f\left(s e^{i \theta}\right)^{p} h(s) s \nu(s) d s\left(-p^{\prime}[h(s)]_{s=r}^{1}\right)^{p-1} \\
& =\left(p^{\prime}\right)^{p-1} \lambda^{-p} h(r)^{p-1} \int_{r}^{1} f\left(s e^{i \theta}\right)^{p} h(s) s \nu(s) d s .
\end{aligned}
$$

This inequality will be repeatedly used throughout the rest of the proof. 
We now proceed to estimate $\int_{E_{\theta}(\lambda)} s \eta(s) d s$. An integration by parts, the assumption $M_{p, \varepsilon}<$ $\infty$ and another integration by parts yield

$$
\begin{aligned}
\int_{a_{k}}^{b_{k}} s \eta(s) d s= & \int_{a_{k}}^{b_{k}} \widehat{\omega}(s)^{p+\varepsilon} \frac{s \eta(s)}{\widehat{\omega}(s)^{p+\varepsilon}} d s \\
= & {\left[\widehat{\omega}(s)^{p+\varepsilon} H(s)\right]_{a_{k}}^{b_{k}}+(p+\varepsilon) \int_{a_{k}}^{b_{k}} \widehat{\omega}(s)^{p+\varepsilon-1} \omega(s) H(s) d s } \\
\leqslant & {\left[\widehat{\omega}(s)^{p+\varepsilon} H(s)\right]_{a_{k}}^{b_{k}}+(p+\varepsilon) M_{p, \varepsilon}^{p} \int_{a_{k}}^{b_{k}} \widehat{\omega}(s)^{p-1} \omega(s) h^{-p}(s) d s } \\
= & {\left[\widehat{\omega}(s)^{p+\varepsilon} H(s)-\frac{(p+\varepsilon)}{p} M_{p, \varepsilon}^{p} \widehat{\omega}(s)^{p} h^{-p}(s)\right]_{a_{k}}^{b_{k}} } \\
& -(p+\varepsilon) M_{p, \varepsilon}^{p} \int_{a_{k}}^{b_{k}} \widehat{\omega}(s)^{p} h^{-p-1}(s) h^{\prime}(s) d s
\end{aligned}
$$

for each $k=1, \ldots, n$. Thus $\int_{E_{\theta}(\lambda)} \eta(s) d s \leqslant S_{1, \theta}+S_{2, \theta}$, where

$$
S_{1, \theta}=\sum_{k=1}^{n}\left[\widehat{\omega}(s)^{p+\varepsilon} H(s)-\frac{(p+\varepsilon)}{p} M_{p, \varepsilon}^{p} \widehat{\omega}(s)^{p} h^{-p}(s)\right]_{a_{k}}^{b_{k}}
$$

and

$$
S_{2, \theta}=-(p+\varepsilon) M_{p, \varepsilon}^{p} \sum_{k=1}^{n} \int_{a_{k}}^{b_{k}} \widehat{\omega}(s)^{p} h^{-p-1}(s) h^{\prime}(s) d s .
$$

The sums $S_{1, \theta}$ and $S_{2, \theta}$ will now be considered separately. Since $H$ is nondecreasing,

$$
\begin{aligned}
& \sum_{k=1}^{n}\left[\widehat{\omega}(s)^{p+\varepsilon} H(s)\right]_{a_{k}}^{b_{k}} \\
& =-\widehat{\omega}\left(a_{1}\right)^{p+\varepsilon} H\left(a_{1}\right)+\sum_{k=1}^{n-1}\left(\widehat{\omega}\left(b_{k}\right)^{p+\varepsilon} H\left(b_{k}\right)-\widehat{\omega}\left(a_{k+1}\right)^{p+\varepsilon} H\left(a_{k+1}\right)\right)+\widehat{\omega}\left(b_{n}\right)^{p+\varepsilon} H\left(b_{n}\right) \\
& \leqslant \sum_{k=1}^{n-1}\left(\widehat{\omega}\left(b_{k}\right)^{p+\varepsilon}-\widehat{\omega}\left(a_{k+1}\right)^{p+\varepsilon}\right) H\left(b_{k}\right)+\widehat{\omega}\left(b_{n}\right)^{p+\varepsilon} H\left(b_{n}\right),
\end{aligned}
$$

where the first negative term was simply discarded. Next, observe that for any $N>M>0$ and $a>0$, the function $g(x)=M\left(a^{N}-x^{N}\right)-N a^{N-M}\left(a^{M}-x^{M}\right)$ is nondecreasing on $[0, a]$. Hence

$$
\widehat{\omega}\left(b_{k}\right)^{p+\varepsilon}-\widehat{\omega}\left(a_{k+1}\right)^{p+\varepsilon} \leqslant \frac{p+\varepsilon}{p} \widehat{\omega}\left(b_{k}\right)^{\varepsilon}\left(\widehat{\omega}\left(b_{k}\right)^{p}-\widehat{\omega}\left(a_{k+1}\right)^{p}\right), \quad k=1, \ldots, n-1 .
$$

This together with (3.5) implies

$$
\begin{aligned}
\sum_{k=1}^{n}\left[\widehat{\omega}(s)^{p+\varepsilon} H(s)\right]_{a_{k}}^{b_{k}} & \leqslant \frac{p+\varepsilon}{p} \sum_{k=1}^{n-1}\left(\widehat{\omega}\left(b_{k}\right)^{p}-\widehat{\omega}\left(a_{k+1}\right)^{p}\right) \widehat{\omega}\left(b_{k}\right)^{\varepsilon} H\left(b_{k}\right)+\widehat{\omega}\left(b_{n}\right)^{p+\varepsilon} H\left(b_{n}\right) \\
& \leqslant M_{p, \varepsilon}^{p} \frac{p+\varepsilon}{p} \sum_{k=1}^{n-1}\left(\widehat{\omega}\left(b_{k}\right)^{p}-\widehat{\omega}\left(a_{k+1}\right)^{p}\right) h^{-p}\left(b_{k}\right)+M_{p, \varepsilon}^{p} \widehat{\omega}\left(b_{n}\right)^{p} h^{-p}\left(b_{n}\right) .
\end{aligned}
$$

By joining this inequality with (3.3), we deduce

$$
S_{1, \theta} \leqslant M_{p, \varepsilon}^{p} \frac{p+\varepsilon}{p}\left(\widehat{\omega}\left(a_{1}\right)^{p} h^{-p}\left(a_{1}\right)+\sum_{k=1}^{n-1}\left(h^{-p}\left(a_{k+1}\right)-h^{-p}\left(b_{k}\right)\right) \widehat{\omega}\left(a_{k+1}\right)^{p}\right) .
$$

Next, use the monotonicity of the auxiliary function $g$ to deduce

$$
h^{-p}\left(a_{k+1}\right)-h^{-p}\left(b_{k}\right) \leqslant p h^{-p+1}\left(a_{k+1}\right)\left[h^{-1}\left(a_{k+1}\right)-h^{-1}\left(b_{k}\right)\right], \quad k=1, \ldots, n-1,
$$


which together with (3.6) and (3.2) yields

$$
\begin{aligned}
S_{1, \theta} \leqslant & M_{p, \varepsilon}^{p} \frac{p+\varepsilon}{p}\left(\widehat{\omega}\left(a_{1}\right)^{p} h^{-p}\left(a_{1}\right)+p \sum_{k=1}^{n-1}\left(h^{-1}\left(a_{k+1}\right)-h^{-1}\left(b_{k}\right)\right) h^{1-p}\left(a_{k+1}\right) \widehat{\omega}\left(a_{k+1}\right)^{p}\right) \\
\leqslant & M_{p, \varepsilon}^{p} \frac{p+\varepsilon}{p}\left(p^{\prime}\right)^{p-1} \lambda^{-p} h^{-1}\left(a_{1}\right) \int_{a_{1}}^{1} f\left(s e^{i \theta}\right)^{p} h(s) s \nu(s) d s \\
& +M_{p, \varepsilon}^{p}(p+\varepsilon)\left(p^{\prime}\right)^{p-1} \lambda^{-p} \sum_{k=1}^{n-1}\left(h^{-1}\left(a_{k+1}\right)-h^{-1}\left(b_{k}\right)\right) \int_{a_{k+1}}^{1} f\left(s e^{i \theta}\right)^{p} h(s) s \nu(s) d s
\end{aligned}
$$

To estimate the sum $S_{2, \theta}$, use (3.2) and integrate by parts to obtain

$$
\begin{aligned}
\frac{S_{2, \theta}}{(p+\varepsilon) M_{p, \varepsilon}^{p}}= & \sum_{k=1}^{n} \int_{a_{k}}^{b_{k}} \widehat{\omega}(s)^{p} h(s)^{-p-1}\left(-h^{\prime}(s)\right) d s \\
\leqslant & \left(p^{\prime}\right)^{p-1} \lambda^{-p} \sum_{k=1}^{n} \int_{a_{k}}^{b_{k}} h(s)^{-2}\left(-h^{\prime}(s)\right)\left(\int_{s}^{1} f\left(s e^{i \theta}\right)^{p} h(t) t \nu(t) d t\right) d s \\
= & \left(p^{\prime}\right)^{p-1} \lambda^{-p} \sum_{k=1}^{n}\left(\left[h(s)^{-1} \int_{s}^{1} f\left(s e^{i \theta}\right)^{p} h(t) t \nu(t) d t\right]_{a_{k}}^{b_{k}}\right. \\
& \left.-\int_{a_{k}}^{b_{k}}\left(-f\left(s e^{i \theta}\right)^{p} h(s) s \nu(s)\right) h(s)^{-1} d s\right) \\
= & \left(p^{\prime}\right)^{p-1} \lambda^{-p} \sum_{k=1}^{n}\left(\left[h(s)^{-1} \int_{s}^{1} f\left(s e^{i \theta}\right)^{p} h(t) t \nu(t) d t\right]_{a_{k}}^{b_{k}}\right. \\
& \left.+\int_{a_{k}}^{b_{k}} f\left(s e^{i \theta}\right)^{p} s \nu(s) d s\right) .
\end{aligned}
$$

By using that $p>1$ and the monotonicity of $h$, and by combining the above inequality with (3.7), it follows that

$$
\begin{aligned}
\frac{\int_{E_{\theta}(\lambda)} s \eta(s) d s}{(p+\varepsilon)\left(p^{\prime}\right)^{p-1} \lambda^{-p} M_{p, \varepsilon}^{p} \leqslant} & \sum_{k=1}^{n} \int_{a_{k}}^{b_{k}} f\left(s e^{i \theta}\right)^{p} s \nu(s) d s+\sum_{k=1}^{n-1} h^{-1}\left(b_{k}\right) \int_{b_{k}}^{a_{k+1}} f\left(s e^{i \theta}\right)^{p} h(s) s \nu(s) d s \\
& +h^{-1}\left(b_{n}\right) \int_{b_{n}}^{1} f\left(s e^{i \theta}\right)^{p} h(s) s \nu(s) d s \\
\leqslant & \sum_{k=1}^{n} \int_{a_{k}}^{b_{k}} f\left(s e^{i \theta}\right)^{p} s \nu(s) d s+\sum_{k=1}^{n-1} \int_{b_{k}}^{a_{k+1}} f\left(s e^{i \theta}\right)^{p} s \nu(s) d s \\
& +\int_{b_{n}}^{1} f\left(s e^{i \theta}\right)^{p} s \nu(s) d s \\
\leqslant & \int_{a_{1}}^{1} f\left(s e^{i \theta}\right)^{p} s \nu(s) d s .
\end{aligned}
$$

By integrating this with respect to $\theta$, we deduce

$$
\eta\left(\left\{z \in \mathbb{D}: T_{\omega}(f)(z) \geqslant \lambda\right\}\right) \lesssim \lambda^{-p} M_{p, \varepsilon}^{p}\|f\|_{L_{\nu}^{p}}^{p}, \quad \lambda>0,
$$

for each non-negative compactly supported step function $f$. It remains to deduce this inequality for arbitrary $f \in L_{\nu}^{p}$. We will accomplish this in a couple steps, starting with simple functions.

Let $\lambda>0$, and let $f \neq 0$ be a non-negative simple function, that is, of the form $f=$ $\sum_{n=1}^{N} \alpha_{n} \chi_{E_{n}}$, where $\alpha_{n} \geqslant 0$ are non-negative constants and $E_{n}$ are disjoint measurable subsets of $\mathbb{D}$. Then, by the proof of [18, Theorem 2.4 p. 71] and [18, Corollary 2.3 p. 71], there exists a 
sequence of non-negative (compactly supported) step functions $\varphi_{k}$ with their range contained in $\left\{\alpha_{n}: n=1, \ldots, N\right\} \cup\{0\}$ and converging to $f$ in $L_{\nu}^{p}$ and pointwise almost everywhere in $\mathbb{D}$. Let $\delta>0$ be arbitrary and denote $M=\max \left\{\alpha_{n}: n=1, \ldots, N\right\}$. Let $R \in(0,1)$ such that $\int_{R}^{1} \eta(s) s d s<\frac{\delta \lambda}{32 M}$. By Egorov's theorem there exists a measurable set $E \subset \mathbb{D}$ such that $\omega_{1}(\mathbb{D} \backslash E)<\frac{\delta \lambda \hat{\omega}(R)}{16 M \int_{0}^{1} \eta(s) s d s}$ and $\varphi_{k} \rightarrow f$ uniformly on $E$, where $\omega_{1}(z)=\frac{\omega(z)}{|z|}$ for $z \in \mathbb{D} \backslash\{0\}$ is a finite measure on $\mathbb{D}$ because $\widehat{\omega}(0)<\infty$. Then

$$
\begin{aligned}
\widehat{\omega}(z)\left|T_{\omega}\left(\varphi_{k}\right)(z)-T_{\omega}(f)(z)\right| \leqslant & \int_{|z|}^{1}\left|\varphi_{k}\left(s \frac{z}{|z|}\right)-f\left(s \frac{z}{|z|}\right)\right| \chi_{E}\left(s \frac{z}{|z|}\right) \omega(s) d s \\
& +2 M \int_{|z|}^{1} \chi_{\mathbb{D} \backslash E}\left(s \frac{z}{|z|}\right) \omega(s) d s
\end{aligned}
$$

for all $z \in \mathbb{D}$. Since $\sup _{z \in \mathbb{D}}\left|\varphi_{k}(z)-f(z)\right| \chi_{E}(z) \rightarrow 0$ as $k \rightarrow \infty$, for fixed $\lambda>0$ there exists $k_{0} \in \mathbb{N}$ such that $\sup _{z \in \mathbb{D}}\left|\varphi_{k}(z)-f(z)\right| \chi_{E}(z)<\frac{\lambda}{4}$ for all $k \geqslant k_{0}$. So, for every point $z \in \mathbb{D}$ such that $\inf _{k \geqslant k_{0}}\left|T_{\omega}\left(\varphi_{k}\right)(z)-T_{\omega}(f)(z)\right|>\lambda / 2$, it follows that

$$
\frac{2 M}{\widehat{\omega}(z)} \int_{|z|}^{1} \chi_{\mathbb{D} \backslash E}\left(s \frac{z}{|z|}\right) \omega(s) d s>\frac{\lambda}{4} .
$$

Thus

$$
\begin{aligned}
\eta\left(\left\{z \in \mathbb{D}:\left|T_{\omega}\left(\varphi_{k}\right)(z)-T_{\omega}(f)(z)\right|>\lambda / 2\right\}\right) & \leqslant \frac{8 M}{\lambda} \int_{\mathbb{D}}\left(\int_{|z|}^{1} \chi_{\mathbb{D} \backslash E}\left(s \frac{z}{|z|}\right) \omega(s) d s\right) \frac{\eta(z)}{\widehat{\omega}(z)} d A(z) \\
& =\frac{8 M}{\lambda} \int_{0}^{1} \omega_{1}((\mathbb{D} \backslash E) \backslash D(0, r)) \frac{r \eta(r)}{\widehat{\omega}(r)} d r
\end{aligned}
$$

for $k \geqslant k_{0}$. Since

$$
\int_{0}^{1} \omega_{1}((\mathbb{D} \backslash E) \backslash D(0, r)) \frac{r \eta(r)}{\widehat{\omega}(r)} d r \leqslant \frac{\omega_{1}(\mathbb{D} \backslash E)}{\widehat{\omega}(R)} \int_{0}^{R} \eta(r) r d r+2 \int_{R}^{1} \eta(r) r d r<\frac{\delta \lambda}{8 M},
$$

we obtain $\eta\left(\left\{z \in \mathbb{D}:\left|T_{\omega}\left(\varphi_{k}\right)(z)-T_{\omega}(f)(z)\right|>\lambda / 2\right\}\right)<\delta$ for $k \geqslant k_{0}$. Then take $\delta=\frac{M_{p, \varepsilon}^{p}\|f\|_{L_{\nu}^{p}}^{p}}{\lambda^{p}}$ and $k_{1} \geqslant k_{0}$ such that $\left\|\varphi_{k}\right\|_{L_{\nu}^{p}} \leqslant 2\|f\|_{L_{\nu}^{p}}$ for all $k \geqslant k_{1}$. Then, bearing in mind (3.8), we deduce

$$
\begin{aligned}
\eta(\{z \in \mathbb{D}: \mid & \left.\left.T_{\omega}(f)(z) \mid \geqslant \lambda\right\}\right) \\
= & \eta\left(\left\{z \in \mathbb{D}:\left|T_{\omega}(f)(z)\right| \geqslant \lambda\right\} \cap\left\{z \in \mathbb{D}:\left|T_{\omega}\left(\varphi_{k}\right)(z)-T_{\omega}(f)(z)\right| \leqslant \lambda / 2\right\}\right) \\
& +\eta\left(\left\{z \in \mathbb{D}:\left|T_{\omega}(f)(z)\right| \geqslant \lambda\right\} \cap\left\{z \in \mathbb{D}:\left|T_{\omega}\left(\varphi_{k}\right)(z)-T_{\omega}(f)(z)\right|>\lambda / 2\right\}\right) \\
\leqslant & \eta\left(\left\{z \in \mathbb{D}:\left|T_{\omega}\left(\varphi_{k}\right)(z)\right| \geqslant \lambda / 2\right\}\right)+\delta \\
\lesssim & M_{p, \varepsilon}^{p} \lambda^{-p}\left\|\varphi_{k}\right\|_{L_{\nu}^{p}}^{p}+\frac{M_{p, \varepsilon}^{p}\|f\|_{L_{\nu}^{p}}^{p}}{\lambda^{p}} \\
\lesssim & M_{p, \varepsilon}^{p} \lambda^{-p}\|f\|_{L_{\nu}^{p}}^{p}
\end{aligned}
$$

for all $k \geqslant k_{1}$. Thus, we deduce that (3.8) holds for simple functions.

Finally, let $f \in L_{\nu}^{p}$ be arbitrary. Since $\left\{z \in \mathbb{D}:\left|T_{\omega}(f)(z)\right|>\lambda\right\} \subset\left\{z \in \mathbb{D}: T_{\omega}(|f|)(z)>\lambda\right\}$, we may assume that $f$ is non-negative. For each $N \in \mathbb{N}$, define $f_{N}=\max \left\{\min \{f, N\}, \frac{1}{N}\right\}$. Then there exists a sequence of simple functions $\varphi_{k}$ such that $N \geqslant \varphi_{k}(z) \geqslant \varphi_{k+1}(z) \geqslant f_{N}(z)$ for all $k \in \mathbb{N}$ and $z \in \mathbb{D}$ and $\varphi_{k} \rightarrow f_{N}$ pointwise in $\mathbb{D}$ as $k \rightarrow \infty$. (Since $1 / f_{N}$ is measurable, there exists an increasing sequence of simple functions $g_{k}$ such that $g_{k}(z) \geqslant \frac{1}{N}$ for all $z \in \mathbb{D}$, and $g_{k} \rightarrow f_{N}$ pointwise in $\mathbb{D}$ as $k \rightarrow \infty$. Take $\varphi_{k}=1 / g_{k}$. See [18, Theorem 4.1 p. 31].) By the dominated convergence theorem there is $k_{2}$ such that $\left\|\varphi_{k}\right\|_{L_{\nu}^{p}} \leqslant 2\left\|f_{N}\right\|_{L_{\nu}^{p}}$ for all $k \geqslant k_{2}$, which 
together with (3.8) yields

$$
\begin{aligned}
\eta\left(\left\{z \in \mathbb{D}: T_{\omega}\left(f_{N}\right)(z)>\lambda\right\}\right) & \leqslant \eta\left(\left\{z \in \mathbb{D}: T_{\omega}\left(\varphi_{k}\right)(z)>\lambda\right\}\right) \\
& \lesssim \lambda^{-p} M_{p, \varepsilon}^{p}\left\|\varphi_{k}\right\|_{L_{\nu}^{p}}^{p} \\
& \lesssim \lambda^{-p} M_{p, \varepsilon}^{p}\left\|f_{N}\right\|_{L_{\nu}^{p}}^{p} \\
& \leqslant \lambda^{-p} M_{p, \varepsilon}^{p}\left(\|f\|_{L_{\nu}^{p}}^{p}+\frac{\nu(\mathbb{D})}{N^{p}}\right), \quad \lambda>0, \quad N \in \mathbb{N} .
\end{aligned}
$$

Since $(\min \{f, N\})_{N \in \mathbb{N}}$ is an increasing sequence of non-negative measurable functions converging pointwise to $f, T_{\omega}(\min \{f, N\})(z) \rightarrow T_{\omega}(f)(z)$ for all $z \in \mathbb{D}$. Then, for fixed $\lambda>0$, by Egorov's theorem there is a measurable set $E \subset \mathbb{D}$ such that $\eta(E)<\lambda^{-p} M_{p, \varepsilon}^{p}\|f\|_{L_{\nu}^{p}}^{p}$ and $N_{0}$ such that $\sup _{z \in \mathbb{D}}\left|T_{\omega}(f)(z)-T_{\omega}(\min \{f, N\})(z)\right| \chi_{\mathbb{D} \backslash E}(z)<\frac{\lambda}{2}$ for all $N \geqslant N_{0}$. Hence

$$
\begin{aligned}
& \eta\left(\left\{z \in \mathbb{D}: T_{\omega}(f)(z)>\lambda\right\}\right) \\
& =\eta\left(\left\{z \in \mathbb{D} \backslash E: T_{\omega}(f)(z)>\lambda\right\}\right)+\eta\left(\left\{z \in E: T_{\omega}(f)(z)>\lambda\right\}\right) \\
& \leqslant \eta\left(\left\{z \in \mathbb{D}: T_{\omega}(\min \{f, N\})(z)>\lambda / 2\right\}\right)+\lambda^{-p} M_{p, \varepsilon}^{p}\|f\|_{L_{\nu}^{p}}^{p} \\
& \leqslant \eta\left(\left\{z \in \mathbb{D}: T_{\omega}\left(f_{N}\right)(z)>\lambda / 2\right\}\right)+\lambda^{-p} M_{p, \varepsilon}^{p}\|f\|_{L_{\nu}^{p}}^{p}, \quad N \geqslant N_{0}, \quad \lambda>0 .
\end{aligned}
$$

Combining this with (3.9) and letting $N \rightarrow \infty$ yields (3.8) for $f \in L_{\nu}^{p}$, and thus (i) is satisfied. This finishes the proof of the theorem.

Proof of Corollary 4. Assume that $T_{\omega}: A_{\nu}^{p} \rightarrow L_{\nu}^{p}$ and $T_{\omega}: L_{\nu}^{p} \rightarrow L_{\nu}^{p, \infty}$ are bounded. Then

$$
\widehat{\mathcal{D}}_{p}(\omega, \nu)=\sup _{0 \leqslant r<1} \frac{\widehat{\omega}(r)^{p}}{\int_{r}^{1} s \nu(s) d s} \int_{0}^{r} \frac{t \nu(t)}{\widehat{\omega}(t)^{p}} d t<\infty
$$

and

$$
M_{p, \varepsilon}(\omega, \nu, \nu)=\sup _{0 \leqslant r<1}\left(\widehat{\omega}(r)^{\varepsilon} \int_{0}^{r} \frac{s \nu(s)}{\widehat{\omega}(s)^{p+\varepsilon}} d s\right)^{\frac{1}{p}}\left(\int_{r}^{1}\left(\frac{\omega(s)}{s \nu(s)}\right)^{p^{\prime}} s \nu(s) d s\right)^{\frac{1}{p^{\prime}}}<\infty
$$

by Theorems 1 and 3. Moreover, (2.13) implies

$$
\frac{\left(\int_{r}^{1} s \nu(s) d s\right)^{\frac{1}{p}}}{\widehat{\omega}(r)} \lesssim\left(\widehat{\omega}(r)^{\varepsilon} \int_{0}^{r} \frac{s \nu(s)}{\widehat{\omega}(s)^{p+\varepsilon}} d s\right)^{\frac{1}{p}} .
$$

Hence

$$
\begin{aligned}
M_{p}(\omega, \nu, \nu) & =\sup _{0 \leqslant r<1}\left(\int_{0}^{r} \frac{s \nu(s)}{\widehat{\omega}(s)^{p}} d s\right)^{\frac{1}{p}}\left(\int_{r}^{1}\left(\frac{\omega(s)}{s \nu(s)}\right)^{p^{\prime}} s \nu(s) d s\right)^{\frac{1}{p^{\prime}}} \\
& \leqslant\left(\widehat{\mathcal{D}}_{p}(\omega, \nu)\right)^{\frac{1}{p}} \sup _{0 \leqslant r<1} \frac{\left(\int_{r}^{1} s \nu(s) d s\right)^{\frac{1}{p}}}{\widehat{\omega}(r)}\left(\int_{r}^{1}\left(\frac{\omega(s)}{s \nu(s)}\right)^{p^{\prime}} s \nu(s) d s\right)^{\frac{1}{p^{\prime}}} \\
& \lesssim\left(\widehat{\mathcal{D}}_{p}(\omega, \nu)\right)^{\frac{1}{p}} \sup _{0 \leqslant r<1}\left(\widehat{\omega}(r)^{\varepsilon} \int_{0}^{r} \frac{s \nu(s)}{\widehat{\omega}(s)^{p+\varepsilon}} d s\right)^{\frac{1}{p}}\left(\int_{r}^{1}\left(\frac{\omega(s)}{s \nu(s)}\right)^{p^{\prime}} s \nu(s) d s\right)^{\frac{1}{p^{\prime}}} \\
& \lesssim\left(\widehat{\mathcal{D}}_{p}(\omega, \nu)\right)^{\frac{1}{p}} M_{p, \varepsilon}(\omega, \nu, \nu) .
\end{aligned}
$$

Thus $T_{\omega}: L_{\nu}^{p} \rightarrow L_{\nu}^{p}$ is bounded. The converse implication is trivial.

Corollary 10. Let $1<p<\infty$. For each $\omega \in \widehat{\mathcal{D}}$ there exists $\nu \in \widehat{\mathcal{D}}$ such that $T_{\omega}: A_{\nu}^{p} \rightarrow L_{\nu}^{p}$ is bounded but $T_{\omega}$ is not bounded from $L_{\nu}^{p}$ to $L_{\nu}^{p, \infty}$. 
Proof. Fix $\omega \in \widehat{\mathcal{D}}$. Take $K>1$ and $r_{n}$ such that $\widehat{\omega}\left(r_{n}\right)=K^{-n} \widehat{\omega}(0)$. Take $t \nu(t)=$ $\omega(t) \sum_{n=0}^{\infty} \chi_{\left[r_{2 n}, r_{2 n+1}\right)}(t)$. Since $\omega$ is not absolutely continuous with respect to $\nu, T_{\omega}$ is not bounded from $L_{\nu}^{p}$ to $L_{\nu}^{p, \infty}$ by Theorem 3 .

On the other hand, since $\int_{t}^{1} s \nu(s) d s=\widehat{\omega}(t)$, it is clear that $\nu \in \widehat{\mathcal{D}}$ and

$$
\int_{0}^{r} \frac{t \nu(t)}{\widehat{\omega}^{p}(t)} d t \leqslant \int_{0}^{r} \frac{\omega(t) d t}{\widehat{\omega}(t)^{p}} \lesssim \frac{1}{\widehat{\omega}(r)^{p-1}}=\frac{\int_{r}^{1} t \nu(t) d t}{\widehat{\omega}(r)^{p}}, \quad 0 \leqslant r<1,
$$

Thus, $T_{\omega}: A_{\nu}^{p} \rightarrow L_{\nu}^{p}$ is bounded by Theorem 1.

We finish the paper providing three weights, $\omega(s)=s, \nu(s)=(1-s)^{p-1}\left(\log \left(\frac{e}{1-s}\right)\right)^{2(p-1)}$ and $\eta(s)=(1-s)^{p-1}\left(\log \left(\frac{e}{1-s}\right)\right)^{p-1}$ such that $T_{\omega}: L_{\nu}^{p} \rightarrow L_{\eta}^{p, \infty}$ is bounded for each $1<p<\infty$ by Theorem 3 , but $T_{\omega}: L_{\nu}^{p} \rightarrow L_{\eta}^{p}$ is unbounded by Theorem

Acknowledgements. We would like to thank professor Francisco J. Martín-Reyes for helpful conversations about Hardy operators and pointing out relevant references on the topic.

\section{REFERENCES}

[1] K. Andersen and B. Muckenhoupt, Weighted weak type inequalities with applications to Hilbert transforms and maximal functions, Studia Math. 72 (1982), no. 1, 9-26.

[2] A. L. Bernardis, F. C. Martín-Reyes and P. Ortega Salvador, Weighted inequalities for Hardy-Steklov Operators, Canad. J. Math. 59 (2007), no. 2 276-295.

[3] J. Duoandikoetxea, F. J. Martín-Reyes, S. Oombrosi, Calderón weights as Muckenhoupt weights, Indiana Univ. Math. J. 62 (2013), no. 3, 891-910.

[4] P. Duren, Theory of $H^{p}$ Spaces, Academic Press, New York-London 1970.

[5] J. Garnett, Bounded analytic functions, Academic Press, New York, 1981.

[6] A. Gogatishvili and J. Lang, The generalized Hilbert operator with kernel and variable integral limits in Banach spaces, J. Inequal. Appl. 4 (1999), no. 1, 1-16.

[7] T. Korhonen, J. A. Peláez and J. Rättyä, Radial two weight inequality for maximal Bergman projection induced by a regular weight, preprint submitted, https://arxiv.org/abs/1805.01256

[8] B. Muckenhoupt, Hardy's inequality with weights, Studia Math.64 (1972), 31-38.

[9] J. A. Peláez, Small weighted Bergman spaces, Proceedings of the summer school in complex and harmonic analysis, and related topics, (2016).

[10] J. Peláez and J. Rättyä, Weighted Bergman spaces induced by rapidly increasing weights, Mem. Amer. Math. Soc. 227 (2014).

[11] J. A. Peláez and J. Rättyä, Generalized Hilbert operators on weighted Bergman spaces, Adv. Math. 240 (2013), 227-267.

[12] J. A. Peláez and J. Rättyä, Embedding theorems for Bergman spaces vis harmonic analysis, Math. Ann. 362 (2015), no. 1-2, 205-239.

[13] J. A. Peláez and J. Rättyä, Two weight inequality for Bergman projection, J. Math. Pures Appl. (9) 105 (2016), no 1, 102-130.

[14] J. A. Peláez, J. Rättyä and K. Sierra, Embedding Bergman spaces into tent spaces, Math. Z. 281 (2015), no. $3,1215-1237$.

[15] J. A. Peláez and J. Rättyä, Bergman projections induced by radial weights, preprint.

[16] F. Pérez-González and J. Rättyä, Derivatives of inner functions in weighted Bergman spaces and the Schwarz-Pick lemma, Proc. Amer. Math. Soc. 145 (2017), no. 5, 2155-2166.

[17] E. Sawyer, Weighted Lebesgue and Lorentz norm inequalities for the Hardy operator. Trans. Amer. Math. Soc. 281 (1984), no. 1, 329-337.

[18] E. M. Stein and R. Shakarchi, Real Analysis. Measure Theory, integration and Hilbert spaces, Princeton Lectures in Analysis, 3. Princeton University Press, Princeton, NJ, 2005. xx+402 pp.

University of Eastern Finland, P.O.Box 111, 80101 Joensuu, Finland

E-mail address: taneli.korhonen@uef.fi

Departamento de Análisis Matemático, Universidad de Málaga, Campus de Teatinos, 29071

MÁlaga, SPAIN

E-mail address: japelaez@uma.es

University of Eastern Finland, P.O.Box 111, 80101 Joensud, Finland

E-mail address: jouni.rattya@uef.fi 